\title{
Cuestionarios de contexto PISA: un estudio sobre los indicadores de evaluación
}

\author{
Background questionnaires of PISA: a study of the assessment indicators
}

\section{González-Such, José; Sancho-Álvarez, Carlos \& Sánchez-Delgado, Purificación}

Universidad de Valencia

\begin{abstract}
Resumen
El sistema de evaluación de PISA ha generado y continúa generando intensos debates sobre su estructura y utilidad. Este artículo se centra en los cuestionarios de contexto, como forma de analizar y entender de manera adecuada los resultados. Los objetivos son analizar los indicadores de contexto que se utilizan en las distintas ediciones de las pruebas PISA, su utilización en distintos estudios y en los resultados de estos estudios. Se proporciona una visión general del modelo utilizado para conseguir que estos indicadores dejen de ser algo que acompaña a las prueba de rendimiento para que alcancen su verdadero sentido: analizar conjuntamente el rendimiento junto con las variables que pueden estar influyendo en los resultados. Como metodología se utiliza el análisis documental de publicaciones relacionadas con PISA y sus resultados, así como un análisis semántico sobre trabajos científicos que ha generado PISA. Los resultados muestran que algunos indicadores se han mantenido a lo largo de las distintas ediciones de PISA, mientras que otros han ido variando. La plasmación de un modelo estable en las ediciones a partir de PISA 2015 en el que se especifican los ítems más relevantes facilitará sin duda el estudio de los resultados a nivel vertical y horizontal. Así, se establece la importancia de los cuestionarios de contexto de PISA para poder entender de manera adecuada sus resultados y la necesidad de realizar estudios más complejos del tipo multinivel o anidados que los que normalmente se utilizan, en general basados en estadísticos descriptivos y/o porcentajes.
\end{abstract}

\section{Palabras clave:}

PISA, cuestionarios de contexto, indicadores de evaluación, evaluación de sistemas educativos, educación, medición.
Fecha de recepción 12 Abril 2016

Fecha de aprobación 16 Junio 2016

Fecha de publicación 16 Junio 2016

\begin{abstract}
The PISA assessment system has generated and continues to generate intense debate about its structure and usefulness. This article focuses on the context questionnaires as a way to analyze and understand the results properly. The objectives are to analyze the context indicators used in the different editions of the PISA tests, used in different studies and the results of these studies. An overview of the model used is provided to ensure that these indicators are no longer something that accompanies the performance test to reach their true meaning: jointly analyze the performance along with the variables that may be influencing the results. As methodology is used document analysis of publications related to PISA and results, as well as a semantic analysis of scientific work that has generated PISA. The results show that some indicators have remained throughout the various editions of PISA, while others have changed. The translation of a stable model in editions from PISA 2015 in which the
\end{abstract}

Reception Date 2016 April 12

\section{Approval Date}

2016 June 16

Publication Date: 2016 June 16 
González-Such, José; Sancho-Álvarez, Carlos \& Sánchez-Delgado, Purificación (2016). Cuestionarios de contexto pisa:

Un estudio sobre los indicadores de evaluación. RELIEVE, 22(1), M7. DOI: http://dx.doi.org/10.7203/relieve.22.1.8429

most relevant items are included will undoubtedly facilitate the study of results at vertical and horizontal level. Thus, the importance of PISA context questionnaires established to properly understand their results and the need for more complex studies of multilevel or nested that normally used, generally based on descriptive statistics and / or percentages.

\section{Keywords:}

PISA, background questionnaires, evaluation indicators, evaluation of educational systems, education, measurement PISA, cuestionarios de contexto, indicadores de evaluación, evaluación de sistemas educativos, educación, medición.

La presencia de PISA en nuestro entorno ya es indiscutible. Las opiniones oscilan entre el apoyo a este sistema y las críticas al mismo (Popkewitz, 2013; Rindermann, 2007). PISA, nacido en el marco de la OCDE como una forma de analizar las diferencias entre países mediante unas pruebas comunes centradas en la medición de las expectativas relacionadas en el rendimiento escolar y sus puntos de referencia o benchmakers (Duru-Bellat, 2013; Popkewitz, 2013), compara la comprensión lectora, la competencia matemática y científica con pruebas alejadas del currículum con el fin de describir la situación de la educación en los países y promover su mejora. Los resultados de estas evaluaciones deberían utilizarse por los países para solucionar sus problemas en materia educativa y para mejorar sus sistemas de enseñanza, y no para compararse con otros en temas de rendimiento (Rendon \& Navarro, 2007). En España la combinación de distintos factores ha hecho que crezca el malestar sobre el sistema educativo (Marchesi, 2006).

Desde el informe Coleman (Coleman, et al., 1966) se ha ido comprobando la relación entre el nivel sociocultural y el rendimiento académico, por lo que es fundamental conocer las condiciones contextuales en que éste ocurre mediante cuestionarios de contexto (ver Sancho-Álvarez, Jornet, \& González-Such, 2016). La evaluación de sistemas educativos tiene algunas debilidades, entre ellas el limitado tratamiento estadístico de las informaciones obtenidas, que podría mejorar mediante el análisis explicativo del producto a partir del contexto, mediante indicadores simples y complejos representados por instrumentos derivados de escalas bien diseñadas. Entre las causas de este limitado tratamiento estadístico se encuentran el limitado conocimiento de algunos modelos explicativos, lo que conduce a analizar los datos de forma descriptiva, o cierto temor a manipular los datos, basado en que el análisis estadístico trastoca la configuración inicial.

A nuestro entender, las mayores dificultades en una evaluación residen en la definición de un modelo teórico que sostenga el sistema y en el abordaje de los indicadores de las variables de entrada, contexto y producto con instrumentos de calidad (López-González, González-Such, \& Lizasoain, 2012, pág. 128).

En general, se considera que las pruebas PISA están metodológicamente bien construidas, aunque quedan cuestiones por resolver, "como la falta de motivación de los alumnos en las evaluaciones sin consecuencias, el rigor en el control de las tasas de respuesta y exclusiones, la equidad y neutralidad en la investigación y el uso e impacto de los resultados” (Martínez Arias, 2006, p. 111).

En PISA 2012 se estableció una estructura que delimitaba un cuerpo de cuestiones que deberían facilitar la comparación entre ciclos para la monitorización de los sistemas educativos (OCDE, 2016). Teniendo en cuenta los objetivos de la evaluación del contexto, las decisiones del Equipo de Gobierno de PISA, el marco general desarrollado por PISA 2012 y recomendaciones de la investigación, PISA 2015 asume que los responsables del Sistema Educativo en los países participantes necesitan ser informados sobre cuatro grandes áreas: resultados, contexto de los estudiantes, procesos de enseñanza aprendizaje y políticas y gobierno educativos (OCDE, 2016).

En el diseño de PISA en general se sostiene el cambio en el enfoque de los cuestionarios de contexto en la evaluación cognitiva: la lectura es el principal dominio de referencia en PISA 2000, 2009 y 2018; matemáticas en PISA 
2003, 2012 y 2021 y ciencias en PISA 2006, 2015 y 2024 (OCDE, 2016).

En la mayoría de los estudios sobre evaluación educativa no se respeta la estructura anidada de los datos PISA, es decir, los alumnos están en un centro, dentro de un distrito, en una ciudad, comunidad autónoma, etc. Como plantean Rendon y Navarro (2007, p. 119),

Sin embargo, multitud de estudios han mostrado cómo factores referentes a variables escolares, del aula y del docente influyen en el logro educativo de los estudiantes (Cervini, 2002, 2003b y 2004; Fernández \& Blanco, 2004; Theule, 2006)

Una solución son los estudios multinivel (Gaviria \& Castro, 2005). No obstante, la investigación sobre las bases de datos PISA es relativamente escasa en nuestro país.

\section{Objetivos}

El presente artículo pretende analizar los indicadores de contexto que se han utilizado en las distintas ediciones de PISA. Como metodología se utilizará el análisis documental basado en las publicaciones relacionadas con PISA y sus resultados. Además, se presenta a nivel global el modelo referido a los ítems de contexto que se quiere introducir en los estudios sobre PISA y se analiza para qué se han utilizado estos ítems en distintos estudios.

\section{Metodología}

A partir de una recopilación de documentos oficiales -informes técnicos y cuestionarios de contexto- se ha realizado un análisis documental (Bisquerra, 2012) para ayudar a complementar y contrastar la información por años.

Los cuestionarios analizados han sido los que se muestran a continuación:

Tabla 1. Fuente de información por países de los cuestionarios

\begin{tabular}{|c|c|c|c|c|c|}
\hline Cuestionarios & 2000 & 2003 & 2006 & 2009 & 2012 \\
\hline Estudiante (ST) & Chile/OECD & México/OECD & México/OECD & España/OECD & México/OECD \\
\hline Centro (SC) & Chile/OECD & México & México/OECD & España/OECD & México/OECD \\
\hline Familia (PA) & & & OECD & México/OECD & México/OECD \\
\hline TIC (IC) & OECD & México/OECD & Chile/OECD & España/OECD & México/OECD \\
\hline
\end{tabular}

Nota: de 2015 aún no se dispone de información completa.

Las fases desarrolladas, de acuerdo a Bisquerra (2012), han sido las siguientes:

1. El rastreo e inventario de los documentos existentes y disponibles.

2. La clasificación de los documentos identificados.
3. La selección de los documentos más pertinentes para los propósitos de la investigación.

4. Una lectura en profundidad del contenido de los documentos seleccionados.

5. Una lectura cruzada y comparativa de los documentos en cuestión (pp.351-352). 


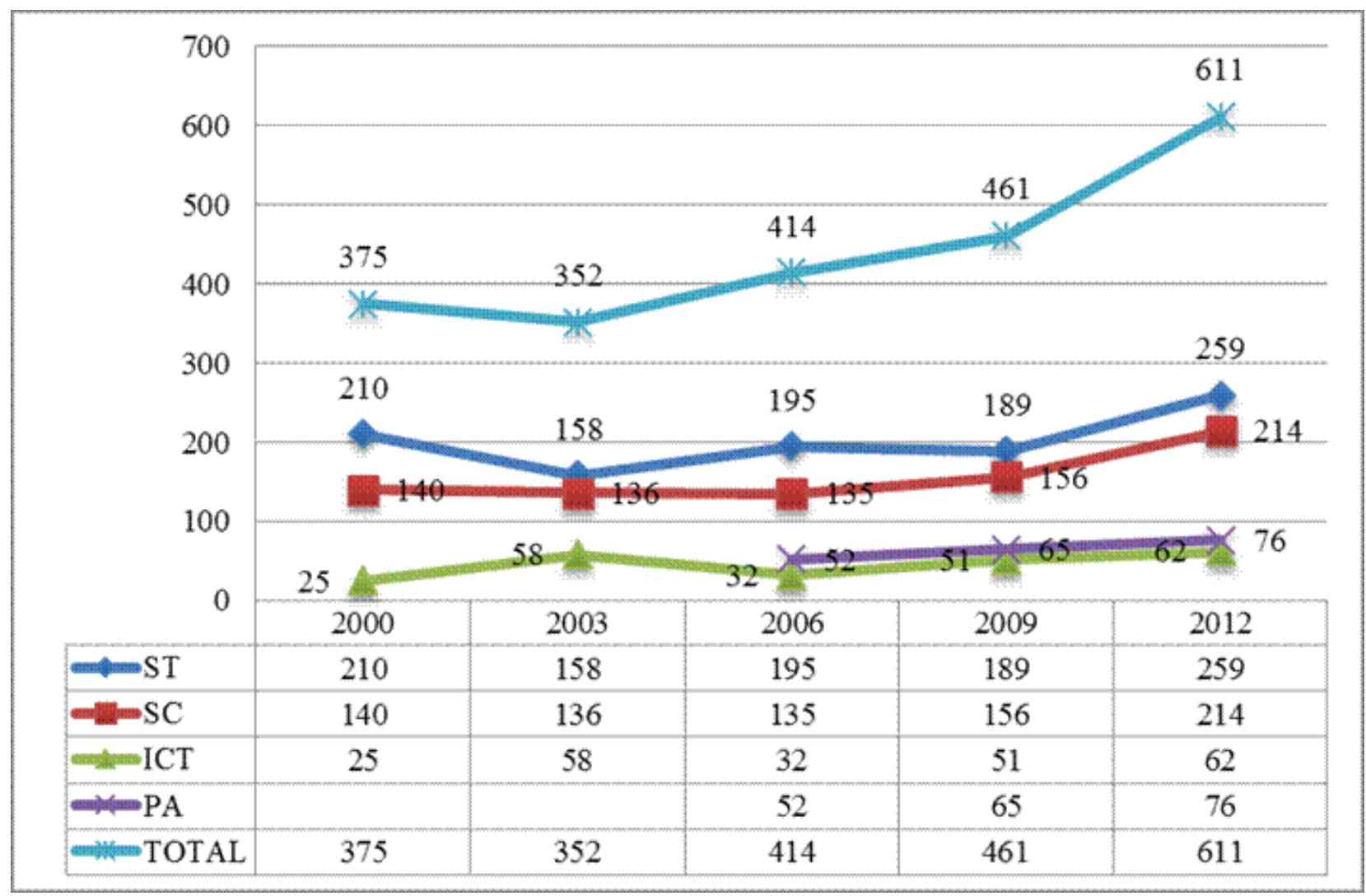

Gráfico 1. Totales ítems por cuestionarios entre años

Encontramos un total de 1011 ítems administrados desde 2000 hasta 2012 para estudiantes, 781 en cuestionarios de centro, 228 sobre TIC, 193 para familias; un total de 2213.

A través del análisis de 2213 ítems entre 32 cuestionarios de contexto entre países (Chile, España y México) y los cuestionarios primarios de contexto oficiales de la OCDE, finalmente se ha trabajado con 964 reactivos estructurados entre 101 indicadores simples y 100 indicadores complejos, de acuerdo a los informe técnicos existentes en la actualidad (Adams \& Wu, 2002, OCDE, 2005, 2009, 2012, 2014) sobre el Programa Internacional de Evaluación de Estudiantes (PISA) 2000, 2003, 2006, 2009 y 2012.

\section{Resultados}

PISA trabaja con dos tipos de indicadores (o índices):

- Indicadores simples: basados en la recodificación directa de las respuestas de una o más variables.
- Indicadores complejos: construidos mediante la aplicación de una metodología de escala que involucra múltiples preguntas y respuestas

Para confirmar teóricamente el comportamiento esperado de los índices y validar su comparabilidad entre los países, utilizan modelos de ecuaciones estructurales. El análisis se realizó utilizando el modelo (SEM) para una confirmación de Análisis Factorial Confirmatorio (CFA) de los ítems del cuestionario (González-Montesinos \& Backhoff, 2010). El CFA se utilizó para validar los índices, y la teoría de respuesta al ítem (TRI) fue usada para producir puntuaciones de la escala. Para los indicadores complejos se escalaron utilizando el modelo de respuesta al ítem de Rasch, y se realizó una estimación de máxima verosimilitud ponderada, indicando los parámetros estimados y delta por cada variable del indicador y por países (Adams \& Wu, 2002).

Por ello, en el presente apartado se describen primeramente los indicadores simples variables (basados en la recodificación 
directa de las respuestas de una o más variables), seguido por los indicadores complejos (que se han construido mediante la aplicación de metodología de escala TRI); indicando para cada indicador las variables que ha utilizado la OCDE para proporcionar estimaciones de los parámetros para cada ítem y que por lo tanto finalmente se ha analizado en profundidad.

\section{Indicadores simples}

Tabla 2. Resultados de Indicadores simples por años

\begin{tabular}{|c|c|c|c|c|c|c|}
\hline \multirow{2}{*}{ Nombre del Indicador PISA } & \multirow{2}{*}{ Acrónimo } & \multicolumn{5}{|c|}{ Año } \\
\hline & & 2000 & 2003 & 2006 & 2009 & 2012 \\
\hline Edad estudiante & AGE & $\mathrm{X}$ & $\mathrm{X}$ & $\mathrm{X}$ & $\mathrm{X}$ & $\mathrm{X}$ \\
\hline Curso estudiante & GRADE & & $\mathrm{X}$ & & $\mathrm{X}$ & $\mathrm{X}$ \\
\hline Programa estudios & PROGN & $\mathrm{X}$ & $\mathrm{X}$ & $\mathrm{X}$ & $\mathrm{X}$ & $\mathrm{X}$ \\
\hline Estructura familiar & FAMSTRUC & $\mathrm{X}$ & $\mathrm{X}$ & & $\mathrm{X}$ & $\mathrm{X}$ \\
\hline Contexto inmigración & INMIG & $\mathrm{X}$ & $\mathrm{X}$ & $\mathrm{X}$ & $\mathrm{X}$ & $\mathrm{X}$ \\
\hline Idioma del hogar & LANGN & $\mathrm{X}$ & $\mathrm{X}$ & $\mathrm{X}$ & $\mathrm{X}$ & $\mathrm{X}$ \\
\hline Orden nacimiento & BRTHORD & $\mathrm{X}$ & & & & \\
\hline Nivel de ocupación más alto de los padres & HISEI & $\mathrm{X}$ & $\mathrm{X}$ & $\mathrm{X}$ & $\mathrm{X}$ & $\mathrm{X}$ \\
\hline Nivel estudios más alto de los padres & PARED & $\mathrm{X}$ & $\mathrm{X}$ & $\mathrm{X}$ & $\mathrm{X}$ & $\mathrm{X}$ \\
\hline Horas de estudio & TOTHRS & $\mathrm{X}$ & & & & \\
\hline Tipo de escuela & SCHLTYPE & $\mathrm{X}$ & $\mathrm{X}$ & $\mathrm{X}$ & $\mathrm{X}$ & $\mathrm{X}$ \\
\hline Tamaño escuela & SCHLSIZE & $\mathrm{X}$ & $\mathrm{X}$ & $\mathrm{X}$ & $\mathrm{X}$ & $\mathrm{X}$ \\
\hline Tamaño clase & CLSIZE & $\mathrm{X}$ & & $\mathrm{X}$ & & $\mathrm{X}$ \\
\hline Tiempo clases & LMNS & $\mathrm{X}$ & $\mathrm{X}$ & & $\mathrm{X}$ & $\mathrm{X}$ \\
\hline Tiempo estudio fuera del centro & OUTHOURS & $\mathrm{X}$ & $\mathrm{X}$ & & & $\mathrm{X}$ \\
\hline Proporción niñas matriculadas centro & PCGIRLS & $\mathrm{X}$ & $\mathrm{X}$ & $\mathrm{X}$ & $\mathrm{X}$ & \\
\hline Disponibilidad de ordenadores & RATCOMP & $\mathrm{X}$ & $\mathrm{X}$ & $\mathrm{X}$ & $\mathrm{X}$ & $\mathrm{X}$ \\
\hline Cantidad personal docente centro & STRATIO & $\mathrm{X}$ & $\mathrm{X}$ & $\mathrm{X}$ & $\mathrm{X}$ & $\mathrm{X}$ \\
\hline Nivel de estudios esperado & SISCED & & $\mathrm{X}$ & & & \\
\hline Situación laboral esperada & BSM & & $\mathrm{X}$ & $\mathrm{X}$ & & $\mathrm{X}$ \\
\hline Selección centro & SELECT & & $\mathrm{X}$ & $\mathrm{X}$ & $\mathrm{X}$ & $\mathrm{X}$ \\
\hline Uso de la evaluación & ASSESS & & $\mathrm{X}$ & & & $\mathrm{X}$ \\
\hline Agrupación por capacidades & ABGROUP & & $\mathrm{X}$ & $\mathrm{X}$ & $\mathrm{X}$ & $\mathrm{X}$ \\
\hline Gestión escolar & AUTRES/AUTCURR & & $\mathrm{X}$ & & & \\
\hline Relaciones inadecuadas estudiante-docente & MSTREL & & $\mathrm{X}$ & & & \\
\hline Ocupación clase obrera o asalariada & HSECATEG & & & $\mathrm{X}$ & $\mathrm{X}$ & \\
\hline Ocupaciones ciencia padres/estudiantes & SCISS & & & $\mathrm{X}$ & & \\
\hline Autonomía centro asignación recursos & RESPRES & & & $\mathrm{X}$ & $\mathrm{X}$ & $\mathrm{X}$ \\
\hline $\begin{array}{llll}\begin{array}{l}\text { Autonomía centro } \\
\text { estudios/evaluación }\end{array} & \text { plan } & \text { de } \\
\end{array}$ & RESPCURR & & & $\mathrm{X}$ & $\mathrm{X}$ & $\mathrm{X}$ \\
\hline Meta-cognición & METASUM & & & & $\mathrm{X}$ & \\
\hline Contexto inmigración de los padres & PQIMMIGF/M & & & & & $\mathrm{X}$ \\
\hline Ciudadanía de los padres & PQCTITZF/M & & & & & $\mathrm{X}$ \\
\hline Repetición curso & REPEAT & & & & & $\mathrm{X}$ \\
\hline & TOTAL & 17 & 22 & 19 & 20 & 23 \\
\hline
\end{tabular}

Como se muestra en la tabla 2, existe diversidad de indicadores simples que van variando según cada oleada de aplicación. Resaltan los indicadores de Gestión escolar y las Relaciones negativas estudiante-docente, que se utilizaron en la aplicación del 2000 y que posteriormente no han sido utilizados. Otros se han ido analizando todos los años como pueden ser Programa de estudios, Contexto inmigración, el nivel más alto de 
ocupación de los padres (HISEI), el nivel más alto de educación de los padres (de acuerdo al ISCED mediante años de escolaridad PARED), Tamaño y Tipo de escuela, Disponibilidad de ordenadores, Cantidad personal docente centro y Agrupación por capacidades. Sin embargo, algunos se han ido incorporando en los últimos años para enriquecer el análisis del contexto, como pueden ser los relativos la Ocupación clase obrera o asalariada, Ocupaciones ciencia padres/estudiantes, Meta-cognición, Contexto inmigración familiar y Ciudadanía familiar; hay que mencionar que otros han ido variando ya que se han convertido junto con otras variables en indicadores complejos, como podremos observar más adelante.

\section{Indicadores complejos}

La metodología de escalamiento y validación de constructo para los indicadores complejos fue usando TRI, con el modelo de un parámetro de Rash (1960-1980) para los ítems dicotómicos, de acuerdo a la siguiente fórmula (OCDE, 2014):

$$
P_{i}\left(\theta_{n}\right)=\frac{\exp \left(\theta_{n}-\delta_{i}\right)}{1+\exp \left(\theta_{n}-\delta_{i}\right)}
$$

Donde $\mathrm{P}_{\mathrm{i}}\left(\theta_{\mathrm{n}}\right)$ es la probabilidad de una persona $n$ de puntuar 1 en el ítem $i, \theta_{\mathrm{n}}$ es el rasgo latente estimado de una persona $n$ y $\delta_{i}$ la localización estimada del ítem $i$ en esta dimensión. Para cada

ítem, las respuestas están modeladas como una función del rasgo latente $\theta_{\mathrm{n}}$ (p. 312)

Para cada ítem, las respuestas están modeladas como una función del rasgo latente qn (p. 312) En el caso de los ítems con más de dos (k) categorías (como por ejemplo con escala tipo Likert) este modelo se puede generalizar para el modelo de crédito parcial CITATION Mas97 \l 3082 (Masters \& Wright, 1997), de acuerdo a la siguiente fórmula (OCDE, 2014):

$$
P_{x_{i}}\left(\theta_{n}\right)=\frac{\exp \sum_{k=0}^{x}\left(\theta_{n}-\delta_{i}+\tau_{i j}\right)}{\sum_{h=0}^{m_{i}} \exp \sum_{k=0}^{h}\left(\theta_{n}-\delta_{i}+\tau_{i k}\right)} x_{i}=0,1, \ldots, m_{i}
$$

Donde $\mathrm{P}_{\mathrm{xj}}\left(\theta_{\mathrm{n}}\right)$ indica la probabilidad de una persona $n$ de puntuar $x$ en un ítem $i$ fuera de las $m$ posibles puntuaciones del ítem. $\theta_{\mathrm{n}}$ indica el rasgo latente de la persona, el parámetro del ítem $\delta_{\mathrm{i}}$ da

la localización del ítem en el continuo latente, y $\tau_{i j}$ indica un parámetro adicional (p. 312

La siguiente tabla contiene indicadores complejos con sus categorías de respuesta para cada aplicación y se indican en cursiva y subrayado los indicadores se repiten idénticos entre años -ver tabla 3-. Asimismo, también indicamos las diferencias entre los indicadores.

Tabla 3. Resultados de Indicadores complejos por años y cálculo de ítems 
González-Such, José; Sancho-Álvarez, Carlos \& Sánchez-Delgado, Purificación (2016). Cuestionarios de contexto pisa: Un estudio sobre los indicadores de evaluación. RELIEVE, 22(1), M7. DOI: http://dx.doi.org/10.7203/relieve.22.1.8429

\begin{tabular}{|c|c|c|c|c|c|c|}
\hline \multirow{2}{*}{$\begin{array}{c}\text { NOMBRE } \\
\text { INDICADOR PISA }\end{array}$} & \multirow{2}{*}{ ACRONIMO } & \multicolumn{5}{|c|}{ Años e ítems analizados en cada escala } \\
\hline & & 2000 & 2003 & 2006 & 2009 & 2012 \\
\hline $\begin{array}{c}\text { Comunicación } \\
\text { cultural }\end{array}$ & CULTCOM & ST19Q01-Q03 & & & & $\begin{array}{l}\text { PA08Q01-Q03 } \\
\text { PA08Q06-Q08 }\end{array}$ \\
\hline $\begin{array}{c}\begin{array}{c}\text { Comunicación } \\
\text { social }\end{array} \\
\end{array}$ & SOCCOM & ST19Q04-Q06 & & & & $\begin{array}{l}\text { PA03Q01-Q04 } \\
\text { PA03Q06-Q09 } \\
\end{array}$ \\
\hline $\begin{array}{c}\text { Apoyo educativo } \\
\text { familiar }\end{array}$ & FAMEDSUP & ST20Q01-Q03 & & & & PA15Q01-Q08 \\
\hline $\begin{array}{c}\text { Actividades } \\
\text { culturales }\end{array}$ & CULTACTV & $\begin{array}{c}\text { ST18Q02 } \\
\text { ST18Q04-Q05 }\end{array}$ & & & & \\
\hline $\begin{array}{l}\text { Riqueza y } \\
\text { posesiones } \\
\text { familiares }\end{array}$ & WEALTH & $\begin{array}{c}\text { ST21Q01-Q04 } \\
\text { ST22Q01-Q02 } \\
\text { ST22Q04 } \\
\text { ST22Q06-Q07 } \\
\end{array}$ & $\begin{array}{c}\text { ST17Q02 } \\
\text { ST17Q04-Q07 }\end{array}$ & $\begin{array}{c}\text { ST13Q02 } \\
\text { ST13Q06 } \\
\text { ST13Q13-Q17 } \\
\text { ST14Q01-Q04 } \\
\end{array}$ & $\begin{array}{c}\text { ST20Q02 } \\
\text { ST20Q06 } \\
\text { ST20Q13-Q17 } \\
\text { ST21Q01-Q05 } \\
\end{array}$ & $\begin{array}{c}\text { ST26Q02 } \\
\text { ST26Q06 } \\
\text { ST26Q14-Q17 } \\
\text { ST27Q01-Q05 } \\
\end{array}$ \\
\hline $\begin{array}{c}\text { Recursos } \\
\text { educativos hogar }\end{array}$ & HEDRES & $\begin{array}{l}\text { ST21Q05-Q08 } \\
\text { ST22Q03 }\end{array}$ & $\begin{array}{c}\text { ST17Q01 } \\
\text { ST17Q03 } \\
\text { ST17Q07 } \\
\text { ST17Q11-Q12 }\end{array}$ & $\begin{array}{c}\text { ST13Q01 } \\
\text { ST13Q03-Q05 } \\
\text { ST13Q07 } \\
\text { ST13Q11-Q12 }\end{array}$ & $\begin{array}{c}\text { ST20Q01 } \\
\text { ST20Q03-Q05 } \\
\text { ST20Q10-Q12 }\end{array}$ & $\begin{array}{c}\text { ST26Q01 } \\
\text { ST26Q03-Q05 } \\
\text { ST26Q10-Q12 }\end{array}$ \\
\hline $\begin{array}{l}\text { Posesiones } \\
\text { culturales }\end{array}$ & CULTPOSS & ST21Q09-Q11 & ST17Q08-Q10 & ST13Q08-Q10 & ST20Q07-Q09 & ST26Q07-Q09 \\
\hline Soporte docente & TEACHSUP & ST26Q05-Q10 & $\begin{array}{l}\text { ST38Q01 } \\
\text { ST38Q03 } \\
\text { ST38Q05 } \\
\text { ST38Q07 } \\
\text { ST38Q10 }\end{array}$ & & & \\
\hline Presión escolar & ACHPRESS & $\begin{array}{c}\text { ST26Q02-Q04 } \\
\text { ST26Q15 } \\
\end{array}$ & & & & \\
\hline Ambiente disciplina & DISCLIM & $\begin{array}{c}\text { ST26Q01 } \\
\text { ST26Q12-Q14 } \\
\text { ST26Q16 } \\
\text { ST26Q17-Q17 }\end{array}$ & $\begin{array}{c}\text { ST38Q02 } \\
\text { ST38Q06 } \\
\text { ST38Q08-Q09 } \\
\text { ST38Q11 } \\
\end{array}$ & & ST36Q01-Q05 & \\
\hline $\begin{array}{c}\text { Relaciones docente- } \\
\text { estudiante }\end{array}$ & $\underline{S T U D R E L}$ & ST30Q01-Q05 & ST26Q01-Q05 & & ST34Q01-Q05 & \\
\hline $\begin{array}{c}\text { Percepciones } \\
\text { discentes sobre la } \\
\text { escuela }\end{array}$ & BELONG & ST31Q01-Q06 & ST27Q01-Q06 & & & \\
\hline Disfrute en lectura & JOYREAD $^{[1]}$ & ST35Q01-Q07 & & ST16Q01-Q05 & ST24Q01-Q11 & \\
\hline Lectura diversa & DIVREAD & ST36Q01-Q06 & & ST25Q01-Q05 & & \\
\hline $\begin{array}{c}\text { Motivación } \\
\text { instrumental }\end{array}$ & INSMOT $^{[2]}$ & $\begin{array}{l}\text { CC01Q06 } \\
\text { CC01Q14 } \\
\text { CC01Q22 }\end{array}$ & $\begin{array}{c}\text { ST30Q02 } \\
\text { ST30Q05 } \\
\text { ST30Q07-Q08 }\end{array}$ & ST35Q01-Q05 & & \\
\hline Interés por lectura & INTREA & $\begin{array}{l}\text { CC02Q06 } \\
\text { CC02Q13 } \\
\text { CC02Q17 }\end{array}$ & & & & \\
\hline $\begin{array}{c}\text { Interés por } \\
\text { matemáticas }\end{array}$ & INTMAT & $\begin{array}{l}\text { CC02Q01 } \\
\text { CC02Q10 } \\
\text { CC02Q21 } \\
\end{array}$ & $\begin{array}{c}\text { ST30Q01 } \\
\text { ST30Q03-Q04 } \\
\text { ST30Q06 } \\
\end{array}$ & & & \\
\hline Interés por ciencia & INTSCIE & & & ST21Q01-Q08 & & \\
\hline $\begin{array}{c}\text { Estrategias de } \\
\text { control }\end{array}$ & $\underline{\text { CSTRAT }^{[3]}}$ & $\begin{array}{l}\text { CC01Q03 } \\
\text { CC01Q13 } \\
\text { CC01Q29 } \\
\text { CC01Q23 } \\
\text { CC01Q27 }\end{array}$ & $\begin{array}{c}\text { ST34Q01 } \\
\text { ST34Q03-Q04 } \\
\text { ST34Q10 } \\
\text { ST34Q12 }\end{array}$ & & $\begin{array}{l}\text { ST27Q02 } \\
\text { ST27Q06 } \\
\text { ST27Q09 } \\
\text { ST27Q11 } \\
\text { ST27Q13 }\end{array}$ & \\
\hline $\begin{array}{l}\text { Estrategias de } \\
\text { memorización }\end{array}$ & $M^{\prime}{ }^{2} R^{[4]}$ & $\begin{array}{l}\text { CC01Q01 } \\
\text { CC01Q05 } \\
\text { CC01Q10 } \\
\text { CC01Q15 }\end{array}$ & $\begin{array}{c}\text { ST34Q06-Q07 } \\
\text { ST34Q09 } \\
\text { ST34Q13 }\end{array}$ & & $\begin{array}{l}\text { ST27Q01 } \\
\text { ST27Q03 } \\
\text { ST27Q05 } \\
\text { ST27Q07 }\end{array}$ & \\
\hline $\begin{array}{l}\text { Estrategías de } \\
\text { aprendizaje }\end{array}$ & ELAB & $\begin{array}{l}\text { CC01Q09 } \\
\text { CC01Q17 } \\
\text { CC01Q21 } \\
\text { CC01Q25 }\end{array}$ & $\begin{array}{l}\text { ST34Q02 } \\
\text { ST34Q05 } \\
\text { ST34Q08 } \\
\text { ST34Q11 } \\
\text { ST34Q14 }\end{array}$ & & $\begin{array}{l}\text { ST27Q04 } \\
\text { ST27Q08 } \\
\text { ST27Q10 } \\
\text { ST27Q12 }\end{array}$ & \\
\hline Esfuerzo y & EFFPER & CC01Q07 & & & & \\
\hline
\end{tabular}


González-Such, José; Sancho-Álvarez, Carlos \& Sánchez-Delgado, Purificación (2016). Cuestionarios de contexto pisa: Un estudio sobre los indicadores de evaluación. RELIEVE, 22(1), M7. DOI: http://dx.doi.org/10.7203/relieve.22.1.8429

\begin{tabular}{|c|c|c|c|c|c|}
\hline perseverancia & & $\begin{array}{l}\text { CC01Q12 } \\
\text { CC01Q20 } \\
\text { CC01Q28 } \\
\end{array}$ & & & \\
\hline $\begin{array}{l}\text { Preferencia por } \\
\text { aprendizaje } \\
\text { cooperativo }\end{array}$ & COOPLRN & $\begin{array}{l}\text { CC02Q02 } \\
\text { CC02Q08 } \\
\text { CC02Q19 } \\
\text { CC02Q22 } \\
\end{array}$ & $\begin{array}{c}\text { ST37Q02 } \\
\text { ST37Q04 } \\
\text { ST37Q06 } \\
\text { ST37Q08-Q09 } \\
\end{array}$ & & \\
\hline $\begin{array}{l}\text { Prerencia por } \\
\text { aprendizaje } \\
\text { competitivo }\end{array}$ & COMPLRN & $\begin{array}{l}\text { CC02Q04 } \\
\text { CC02Q11 } \\
\text { CC02Q16 } \\
\text { CC02Q24 }\end{array}$ & $\begin{array}{l}\text { ST37Q01 } \\
\text { ST37Q03 } \\
\text { ST37Q05 } \\
\text { ST37Q07 } \\
\text { ST37Q10 } \\
\end{array}$ & & \\
\hline $\begin{array}{c}\text { Auto-concepto en } \\
\text { lectura }\end{array}$ & SCVERB & $\begin{array}{l}\text { CC02Q05 } \\
\text { CC02Q09 } \\
\text { CC02Q23 } \\
\end{array}$ & & & \\
\hline $\begin{array}{l}\text { Auto-concepto en } \\
\text { matemáticas }\end{array}$ & SCMAT $^{[5]}$ & $\begin{array}{l}\text { CC02Q12 } \\
\text { CC02Q15 } \\
\text { CC02Q18 }\end{array}$ & $\begin{array}{c}\text { ST32Q02 } \\
\text { ST32Q04 } \\
\text { ST32Q06-Q07 } \\
\text { ST32Q09 } \\
\end{array}$ & ST37Q01-Q06 & \\
\hline $\begin{array}{l}\text { Auto-concepto } \\
\text { académico }\end{array}$ & SCACAD & $\begin{array}{l}\text { CC02Q03 } \\
\text { CC02Q07 } \\
\text { CC02Q20 } \\
\end{array}$ & & & \\
\hline $\begin{array}{c}\text { Auto-eficacia } \\
\text { percibida }\end{array}$ & SELFEF ${ }^{[6]}$ & $\begin{array}{l}\text { CC01Q02 } \\
\text { CC01Q18 } \\
\text { CC01Q22 } \\
\end{array}$ & ST31Q01-Q08 & ST17Q01-Q08 & \\
\hline $\begin{array}{c}\text { Control de } \\
\text { expectativas }\end{array}$ & CEXP & $\begin{array}{l}\text { CC01Q04 } \\
\text { CC01Q11 } \\
\text { CC01Q16 } \\
\text { CC01Q24 } \\
\end{array}$ & & & \\
\hline $\begin{array}{c}\text { Competencia digital } \\
\text { percibida }\end{array}$ & COMAB & $\begin{array}{c}\text { IT02Q01-Q03 } \\
\text { IT03Q01 } \\
\end{array}$ & & & \\
\hline $\begin{array}{l}\text { Confianza en la } \\
\text { realización de } \\
\text { tareas diarias }\end{array}$ & ROUTCONF & & $\begin{array}{c}\text { IC06Q01 } \\
\text { IC06Q03-Q05 } \\
\text { IC06Q07-Q11 } \\
\text { IC06Q18 } \\
\text { IC06Q21 } \\
\end{array}$ & & \\
\hline Uso TIC & PRGUSE[7] & $\begin{array}{l}\text { IT05Q03-Q04 } \\
\text { IT06Q02-Q05 }\end{array}$ & $\begin{array}{c}\text { IC05Q03 } \\
\text { IC05Q05 } \\
\text { IC05Q07-Q09 } \\
\text { IC05Q11 } \\
\end{array}$ & $\begin{array}{c}\text { IC04Q03 } \\
\text { IC04Q05 } \\
\text { IC04Q07-Q08 } \\
\text { IC04Q10 } \\
\end{array}$ & IC06Q01-Q09 \\
\hline $\begin{array}{l}\text { Confianza en la } \\
\text { realización de } \\
\text { tareas digitales }\end{array}$ & INTCONF & & $\begin{array}{l}\text { IC06Q12-Q14 } \\
\text { IC06Q19 } \\
\text { IC06Q22 }\end{array}$ & $\begin{array}{c}\text { IC05Q01 } \\
\text { IC05Q07-Q09 } \\
\text { IC05Q13 } \\
\text { IC05Q15 } \\
\end{array}$ & \\
\hline $\begin{array}{c}\text { Confianza en la } \\
\text { realización de } \\
\text { tareas digitales de } \\
\text { alto nivel } \\
\end{array}$ & HIGHCONF & & $\begin{array}{l}\text { IC06Q02 } \\
\text { IC06Q06 } \\
\text { IC06Q15-Q17 } \\
\text { IC06Q23 } \\
\end{array}$ & $\begin{array}{c}\text { IC05Q02-Q04 } \\
\text { IC05Q10-Q12 } \\
\text { IC05Q14 } \\
\text { IC05Q16 } \\
\end{array}$ & IC08Q01-Q05 \\
\hline $\begin{array}{c}\text { Uso TIC para } \\
\text { entretenimiento }\end{array}$ & INTUSE & & $\begin{array}{c}\text { IC05Q01-Q02 } \\
\text { IC05Q04 } \\
\text { IC05Q06 } \\
\text { IC05Q10 } \\
\text { IC05Q12 } \\
\end{array}$ & $\begin{array}{c}\text { IC04Q01-Q02 } \\
\text { IC04Q04 } \\
\text { IC04Q06 } \\
\text { IC04Q09 } \\
\text { IC04Q11 } \\
\end{array}$ & IC04Q01-Q09 \\
\hline $\begin{array}{c}\text { Actitudes hacia } \\
\text { ordenadores }\end{array}$ & ATTCOMP & $\begin{array}{l}\text { IT07Q01 } \\
\text { IT08Q01 } \\
\text { IT09Q01 } \\
\text { IT10Q01 } \\
\end{array}$ & IC07Q01-Q04 & & IC10Q01-Q04 \\
\hline $\begin{array}{c}\text { Autonomía de } \\
\text { centro }\end{array}$ & SCHAUTON & SC22Q01-Q12 & SC26Q01-Q12 & & SC24Q01-Q12 \\
\hline $\begin{array}{c}\begin{array}{c}\text { Participación } \\
\text { docente }\end{array} \\
\end{array}$ & $\underline{\text { TCHPARTI }}$ & SC22Q01-Q12 & SC26Q01-Q12 & & SC24Q01-Q12 \\
\hline $\begin{array}{l}\text { Factores docentes } \\
\text { que afectan al clima } \\
\text { escolar }\end{array}$ & TEACBEHA & $\begin{array}{c}\text { SC19Q01 } \\
\text { SC19Q03 } \\
\text { SC19Q07-Q08 }\end{array}$ & $\begin{array}{c}\text { ST25Q03 } \\
\text { ST25Q05-Q06 } \\
\text { ST25Q09 }\end{array}$ & & $\begin{array}{c}\text { SC17Q01 } \\
\text { SC17Q03 } \\
\text { SC17Q05-Q06 }\end{array}$ \\
\hline
\end{tabular}


González-Such, José; Sancho-Álvarez, Carlos \& Sánchez-Delgado, Purificación (2016). Cuestionarios de contexto pisa: Un estudio sobre los indicadores de evaluación. RELIEVE, 22(1), M7. DOI: http://dx.doi.org/10.7203/relieve.22.1.8429

\begin{tabular}{|c|c|c|c|c|c|c|}
\hline & & $\begin{array}{l}\text { SC19Q15 } \\
\text { SC19Q13 } \\
\text { SC17Q16 } \\
\end{array}$ & $\begin{array}{l}\text { ST25Q11 } \\
\text { ST25Q13 }\end{array}$ & & & $\begin{array}{l}\text { SC17Q09 } \\
\text { SC17Q11 } \\
\text { SC17Q13 } \\
\end{array}$ \\
\hline $\begin{array}{l}\text { Factores discentes } \\
\text { que afectan al clima } \\
\text { escolar }\end{array}$ & $\underline{\text { STUDBEHA }}$ & $\begin{array}{c}\text { SC19Q02 } \\
\text { SC19Q06 } \\
\text { SC19Q09-Q10 } \\
\text { SC19Q13 } \\
\text { SC19Q15 } \\
\end{array}$ & $\begin{array}{c}\text { ST25Q02 } \\
\text { ST25Q04 } \\
\text { ST25Q07-Q08 } \\
\text { ST25Q10 } \\
\text { ST25Q12 } \\
\end{array}$ & & & $\begin{array}{c}\text { SC17Q02 } \\
\text { SC17Q04 } \\
\text { SC17Q07-Q08 } \\
\text { SC17Q10 } \\
\text { SC17Q12 } \\
\end{array}$ \\
\hline Moral docente & TCMORALE & SC20Q01-Q04 & SC24Q01-Q04 & & & \\
\hline $\begin{array}{l}\text { Calidad recursos } \\
\text { educativos centro }\end{array}$ & $\underline{\text { SCMATEDU }}{ }^{[8]}$ & SC11Q04-Q09 & $\begin{array}{c}\text { SC08Q09 } \\
\text { SC08Q15-Q20 } \\
\end{array}$ & SC14Q07-Q13 & SC11Q07-Q13 & \\
\hline $\begin{array}{c}\text { Calidad } \\
\text { infraestructuras } \\
\text { centro } \\
\end{array}$ & $\underline{S C M A T B U I}$ & SC11Q01-Q03 & SC08Q11-Q13 & & & \\
\hline $\begin{array}{c}\text { Escasez de } \\
\text { profesorado } \\
\end{array}$ & $\underline{\text { TCSHORT }^{[9]}}$ & SC21Q01-Q04 & SC08Q01-Q06 & SC14Q01-Q04 & & SC11Q01-Q04 \\
\hline $\begin{array}{c}\text { Posesiones del } \\
\text { hogar }\end{array}$ & HOMEPOS & & $\begin{array}{l}\text { ST17Q02-Q12 } \\
\text { ST19Q01 }\end{array}$ & $\begin{array}{l}\text { ST13Q01-Q17 } \\
\text { ST14Q01-Q04 } \\
\text { ST15Q01 }\end{array}$ & $\begin{array}{l}\text { ST20Q01-Q17 } \\
\text { ST21Q01-Q05 } \\
\text { ST22Q01 }\end{array}$ & $\begin{array}{c}\text { ST26Q01-Q12 } \\
\text { ST26Q14-Q17 } \\
\text { ST27Q01-Q05 } \\
\text { ST28Q01 } \\
\end{array}$ \\
\hline $\begin{array}{l}\text { Índice de estatus } \\
\text { económico-social y } \\
\text { cultural }\end{array}$ & $\underline{E S C S^{[10]}}$ & $\begin{array}{c}\text { HISEI } \\
\text { PARED } \\
\text { WEALTH } \\
\text { HEDRES } \\
\text { CULTPOSS } \\
\end{array}$ & $\begin{array}{c}\text { HISEI } \\
\text { PARED } \\
\text { HOMEPOS }\end{array}$ & $\begin{array}{c}\text { HISEI } \\
\text { PARED } \\
\text { HOMEPOS }\end{array}$ & $\begin{array}{c}\text { HISEI } \\
\text { PARED } \\
\text { HOMEPOS }\end{array}$ & $\begin{array}{c}\text { HISEI } \\
\text { PARED } \\
\text { HOMEPOS }\end{array}$ \\
\hline $\begin{array}{c}\text { Actitudes hacia la } \\
\text { escuela }\end{array}$ & $\underline{A T S C H L}$ & & ST24Q01-Q04 & & ST33Q01-Q04 & \\
\hline $\begin{array}{l}\text { Ansiedad en } \\
\text { matemáticas }\end{array}$ & ANXMAT & & $\begin{array}{l}\text { ST32Q01 } \\
\text { ST32Q03 } \\
\text { ST32Q05 } \\
\text { ST32Q08 } \\
\text { ST32Q10 } \\
\end{array}$ & & & \\
\hline Moral discente & STMORALE & & SC11Q01-Q07 & & & \\
\hline $\begin{array}{c}\text { Colegialidad } \\
\text { docente }\end{array}$ & TCCONS & & $\begin{array}{l}\text { ST21Q03 } \\
\text { ST22Q03 } \\
\text { ST23Q03 } \\
\end{array}$ & & & \\
\hline $\begin{array}{c}\text { Motivación futura } \\
\text { ciencias }\end{array}$ & SCIEFUT & & & ST29Q01-Q04 & & \\
\hline $\begin{array}{c}\text { Preparación escolar } \\
\text { para carreras } \\
\text { ciencias }\end{array}$ & CARPREP & & & ST27Q01-Q04 & & \\
\hline $\begin{array}{c}\text { Valor general } \\
\text { ciencias }\end{array}$ & GENSCIE & & & $\begin{array}{c}\text { ST18Q01-Q02 } \\
\text { ST18Q04 } \\
\text { ST18Q06 } \\
\end{array}$ & & \\
\hline $\begin{array}{c}\text { Percepción familiar } \\
\text { importancia } \\
\text { ciencias } \\
\end{array}$ & PQSCIMP & & & PA04Q01-Q04 & & \\
\hline $\begin{array}{l}\text { Percepción familiar } \\
\text { valor general } \\
\text { ciencias }\end{array}$ & PQGENSCI & & & $\begin{array}{c}\text { PA06Q01-Q02 } \\
\text { PA06Q04 } \\
\text { PA06Q06 } \\
\text { PA06Q09 } \\
\end{array}$ & & \\
\hline $\begin{array}{c}\text { Actividades } \\
\text { matemáticas centro }\end{array}$ & MACTIV & SCQ17Q01-Q05 & & ST19Q01-Q06 & & $\begin{array}{c}\text { SC21 } \\
\text { SC16Q05-Q06 } \\
\text { SC16Q08 } \\
\end{array}$ \\
\hline $\begin{array}{l}\text { Valor personal } \\
\text { ciencias }\end{array}$ & PERSCIE & & & $\begin{array}{c}\text { ST18Q03 } \\
\text { ST18Q05 } \\
\text { ST18Q07-Q08 } \\
\text { ST18Q10 } \\
\end{array}$ & & \\
\hline $\begin{array}{c}\text { Percepción } \\
\text { personal familiar } \\
\text { valor ciencias } \\
\end{array}$ & PQPERSCI & & & $\begin{array}{c}\text { PA06Q03 } \\
\text { PA06Q05 } \\
\text { PA06Q07-Q08 } \\
\end{array}$ & & \\
\hline $\begin{array}{l}\text { Sensibilidad } \\
\text { problemática }\end{array}$ & ENVAWARE & & & ST22Q01-Q05 & & \\
\hline
\end{tabular}


González-Such, José; Sancho-Álvarez, Carlos \& Sánchez-Delgado, Purificación (2016). Cuestionarios de contexto pisa: Un estudio sobre los indicadores de evaluación. RELIEVE, 22(1), M7. DOI: $\underline{\text { http://dx.doi.org/10.7203/relieve.22.1.8429 }}$

ambiental

\begin{tabular}{|c|c|c|c|c|}
\hline $\begin{array}{c}\text { Percepción } \\
\text { problemática } \\
\text { ambiental }\end{array}$ & ENVPERC & ST24Q01-Q06 & & \\
\hline $\begin{array}{c}\text { Percepción familiar } \\
\text { problemática } \\
\text { ambiental } \\
\end{array}$ & PQENPERC & PA07Q01-Q06 & & \\
\hline $\begin{array}{c}\text { Optimismo } \\
\text { ambiental }\end{array}$ & ENVOPT & ST25Q01-Q06 & & \\
\hline $\begin{array}{c}\text { Optimismo familiar } \\
\text { ambiental }\end{array}$ & $\overline{P Q E N V O P T}$ & PA08Q01-Q06 & & \\
\hline $\begin{array}{c}\begin{array}{c}\text { Responsabilidad } \\
\text { desarrollo } \\
\text { sostenible }\end{array} \\
\end{array}$ & RESPDEV & ST26Q01-Q07 & & \\
\hline $\begin{array}{c}\text { Información } \\
\text { discentes carreras } \\
\text { ciencias }\end{array}$ & CARINFO & ST28Q01-Q04 & & \\
\hline $\begin{array}{l}\text { Enseñanza ciencias: } \\
\text { interacción }\end{array}$ & SCINTACT $^{[12]}$ & $\begin{array}{l}\text { ST34Q01 } \\
\text { ST34Q05 } \\
\text { ST34Q09 } \\
\text { ST34Q13 } \\
\end{array}$ & ST37Q01-Q07 & \\
\hline $\begin{array}{c}\text { Enseñanza ciencias: } \\
\text { prácticas }\end{array}$ & SCHANDS ${ }^{[13]}$ & $\begin{array}{c}\text { ST34Q02-Q03 } \\
\text { ST34Q06 } \\
\text { ST34Q14 } \\
\end{array}$ & $\begin{array}{l}\text { ST38Q01-Q08 } \\
\text { ST38Q01 }\end{array}$ & \\
\hline $\begin{array}{c}\text { Enseñanza ciencias: } \\
\text { proyectos }\end{array}$ & SCINVEST & $\begin{array}{l}\text { ST34Q08 } \\
\text { ST34Q11 } \\
\text { ST34Q16 } \\
\end{array}$ & & \\
\hline $\begin{array}{l}\text { Enseñanza ciencias: } \\
\text { modelos aplicados }\end{array}$ & SCAPPLY & $\begin{array}{l}\text { ST34Q07 } \\
\text { ST34Q12 } \\
\text { ST34Q15 } \\
\text { ST34Q17 } \\
\end{array}$ & & \\
\hline $\begin{array}{c}\text { Actividades } \\
\text { escolares } \\
\text { promoción ciencias }\end{array}$ & SCIPROM & SC20Q01-Q05 & & \\
\hline $\begin{array}{c}\text { Actividades } \\
\text { escolares } \\
\text { promoción temas } \\
\text { ambientales } \\
\end{array}$ & ENVLEARN & SC22Q01-Q05 & & \\
\hline $\begin{array}{c}\text { Actividades ciencias } \\
\text { a los } 10 \text { años } \\
\end{array}$ & PQSCIACT & PA02Q01-Q05 & & \\
\hline $\begin{array}{l}\text { Percepción familiar } \\
\text { calidad centro }\end{array}$ & $\underline{P Q S C H O O L}$ & PA03Q01-Q07 & & PA14Q01-Q07 \\
\hline $\begin{array}{c}\text { Información } \\
\text { familiar carreras } \\
\text { ciencias }\end{array}$ & PQSCCAR & PA05Q02-Q05 & & \\
\hline $\begin{array}{c}\begin{array}{c}\text { Disponibilidad TIC } \\
\text { hogar }\end{array} \\
\end{array}$ & ST17Q04-Q06 & & ST20Q05-Q06 & IC01Q01-Q08 \\
\hline Lectura digital & ONLNREAD & & ST26Q01-Q07 & \\
\hline Uso librerías & LIBUSE & & ST39Q01-Q07 & \\
\hline $\begin{array}{c}\text { Disponibilidad TIC } \\
\text { centro }\end{array}$ & ICTSCH & & & IC02Q01-Q05 \\
\hline $\begin{array}{c}\text { Uso TIC en casa } \\
\text { para tareas } \\
\text { escolares }\end{array}$ & HOMESCH & & & IC05Q01-Q05 \\
\hline $\begin{array}{l}\text { Actividades extra- } \\
\text { escolares centro }\end{array}$ & EXCURACT & & & SC13Q01-Q13 \\
\hline Liderazgoo escolar & LDRSHP & & & SC26Q01-Q14 \\
\hline $\begin{array}{l}\text { Motivación por la } \\
\text { lectura en el hogar }\end{array}$ & MOTREAD & & & PA06Q01-Q04 \\
\hline $\begin{array}{l}\text { Recursos para la } \\
\text { lectura en el hogar }\end{array}$ & READRES & & & PA07Q01-Q06 \\
\hline
\end{tabular}


Destacan los indicadores complejos de Autoconcepto Académico, Control de Expectativas, Actividades Culturales, Presión académica, Interés en lectura, Esfuerzo y perseverancia, Autoconcepto en lectura $y$ Competencia digital percibida que únicamente se han analizado en el 2000, así como Confianza en la realización de tareas diarias, Ansiedad en matemáticas, Moral discente y Colegialidad docente en el 2003. Asimismo, destacan multitud de indicadores muy interesantes con respecto a ciencias y el medio ambiente, pero que solo ha sido tan amplio en el año 2006, como se puede observar en la tabla 3; desde Motivación futura ciencias o Valor general ciencias has Actividades escolares promoción ciencias. En este sentido, durante los años 2009 y 2012 se amplían los indicadores con respecto a tecnologías y competencia digital.

En general, podemos observar que los indicadores complejos a través de los años se focalizan en diferentes temas centrales. Por ejemplo, en PISA 2000 y PISA 2009 la atención se centra en Matemáticas, en PISA 2003 y PISA 2012 es la Lectura el foco, y en PISA 2006 son las Ciencias; También en PISA 2012 existen muchos indicadores centrados en la competencia digital.

Por todo ello, como se muestra en el siguiente gráfico, de manera analítica podemos ir observando la variación de variables estudiadas, así como su evolución en cuanto a tendencias por oleadas PISA según el tipo de indicador -simple SI o complejo CI- junto con el total conjunto.

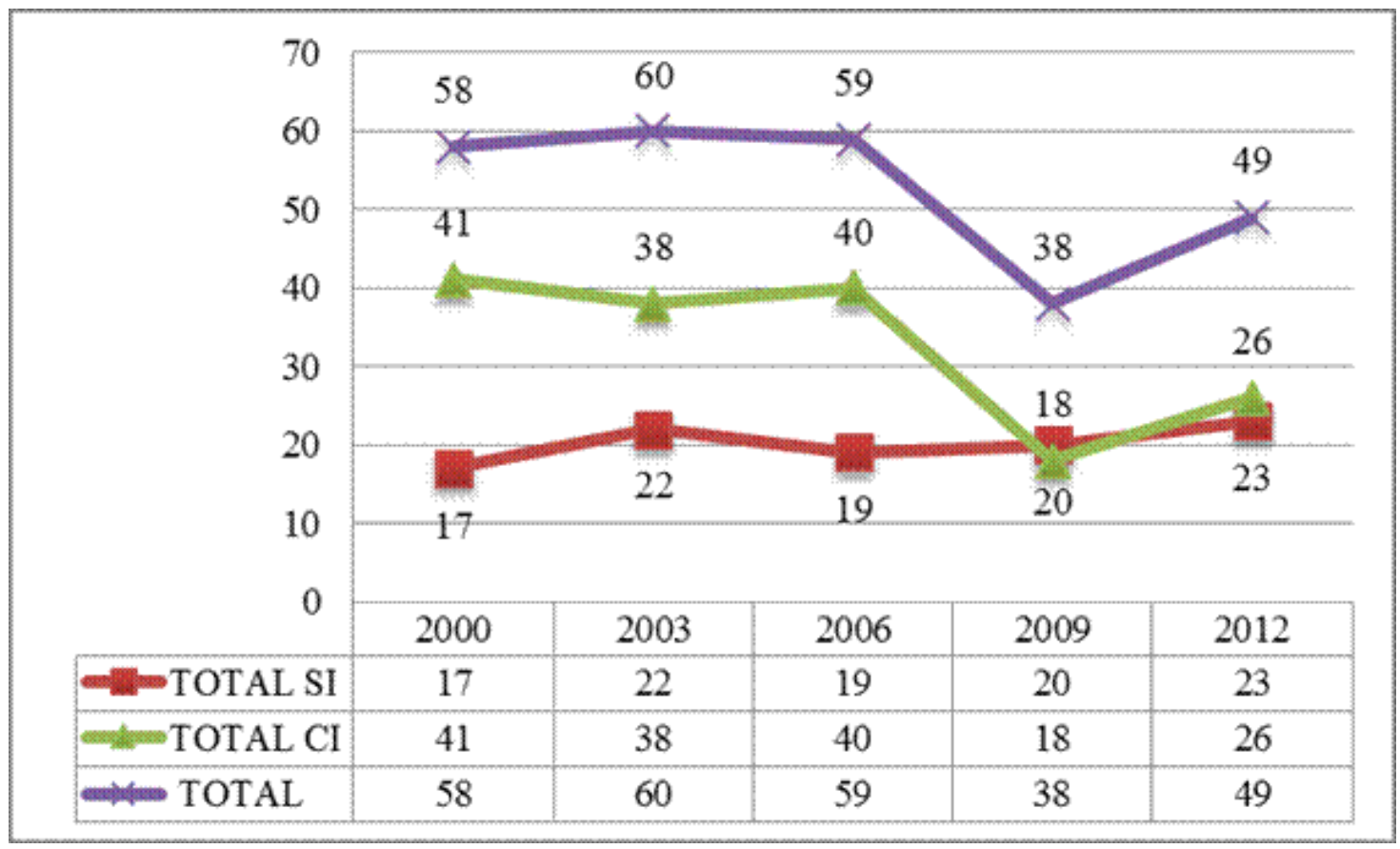

Gráfico 2. Totales por tipo indicador

La evolución va creciendo en cuanto a indicadores simples pero va siendo menor en relación a indicadores complejos. Asimismo, encontramos un total de 101 indicadores simples y 100 indicadores complejos (un total de 201 indicadores analizados -ver tabla $1 \mathrm{y}$ 2). 


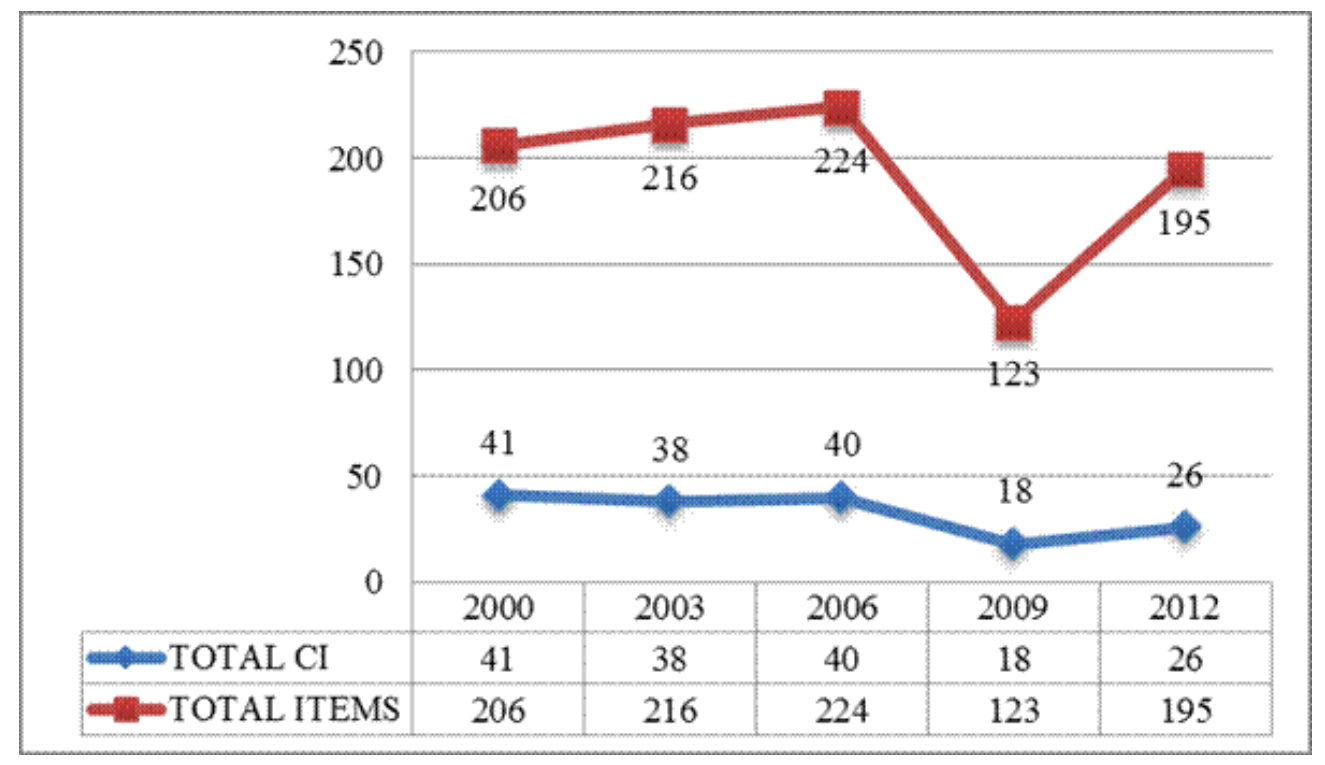

Gráfico 3. Totales indicadores complejos e ítems

Si observamos en profundidad el número de indicadores complejos (TOTAL CI) y sus respectivos items asociados para medir el conjunto por aplicaciones (TOTAL ITEMS), podemos ver que la tendencia es en cierta medida decreciente. Asimismo, en relación a los 100 indicadores complejos se han estudiado un total de 964 variables asociadas (ver tabla 3).

Sin embargo, aunque el número de indicadores complejos sea cada vez menor (gráfico 3), los ítems que se incluyen por cuestionarios va siendo mayor por año -ver gráfco 1-; al igual que pasaba en relación a los indicadores simples (gráfico 2).

\section{EI caso del estatus socio-económico y cultural (ESCS)}

El indicador ESCS fue usado por primera vez en PISA 2000 mediante un análisis que fue derivado desde cinco indicadores: el nivel más alto de ocupación de los padres (HISEI), el nivel más alto de educación de los padres (de acuerdo al ISCED mediante años de escolaridad PARED), riqueza de la familia
(WEALTH), posesiones culturales (CULTPOSS) y los recursos educativos del hogar (HEDRES).

El ESCS para PISA 2003 y 2006 fue derivado desde tres indicadores relacionados con el contexto familiar: el nivel más alto de ocupación de los padres (HISEI), el nivel más alto de educación de los padres (de acuerdo al ISCED mediante años de escolaridad), y las posesiones del hogar incluyendo el número de libros en casa (HOMEPOS).

Por esta razón, en PISA 2003, PISA 2006, PISA 2009 y PISA 2012 las variables comprimidas para el indicador de posesiones del hogar (HOMEPOS) son todos los ítems de los indicadores WEALTH, CULTPOSS y HEDRES, así como también los libros en casa (pregunta específica estructurada en una escala de respuesta de cuatro puntos; menos o igual a 25 libros, 26-100 libros, 101-500 libros, más de 500 libros).

Para cada país, las puntuaciones del ESCS fueron obtenidas mediante la siguiente fórmula (OCDE, 2014):

$$
E S C S=\frac{\beta_{1} H_{I S E I}+\beta_{2} P A R E D^{\prime}+\beta_{3} \text { HOMEPOS }^{\prime}}{\varepsilon_{f}}
$$

Donde $\beta, \beta 1$ y $\beta 2$ el peso de los factores OCDE; HISEI', PARED' y HOMEPOS' las variables

"OCDE estandarizadas" y ci es el valor propio del primer componente principal (p.352) 
González-Such, José; Sancho-Álvarez, Carlos \& Sánchez-Delgado, Purificación (2016). Cuestionarios de contexto pisa: Un estudio sobre los indicadores de evaluación. RELIEVE, 22(1), M7. DOI: http://dx.doi.org/10.7203/relieve.22.1.8429

Tabla 4. Resultados del cálculo de ECSC sobre ítems y años

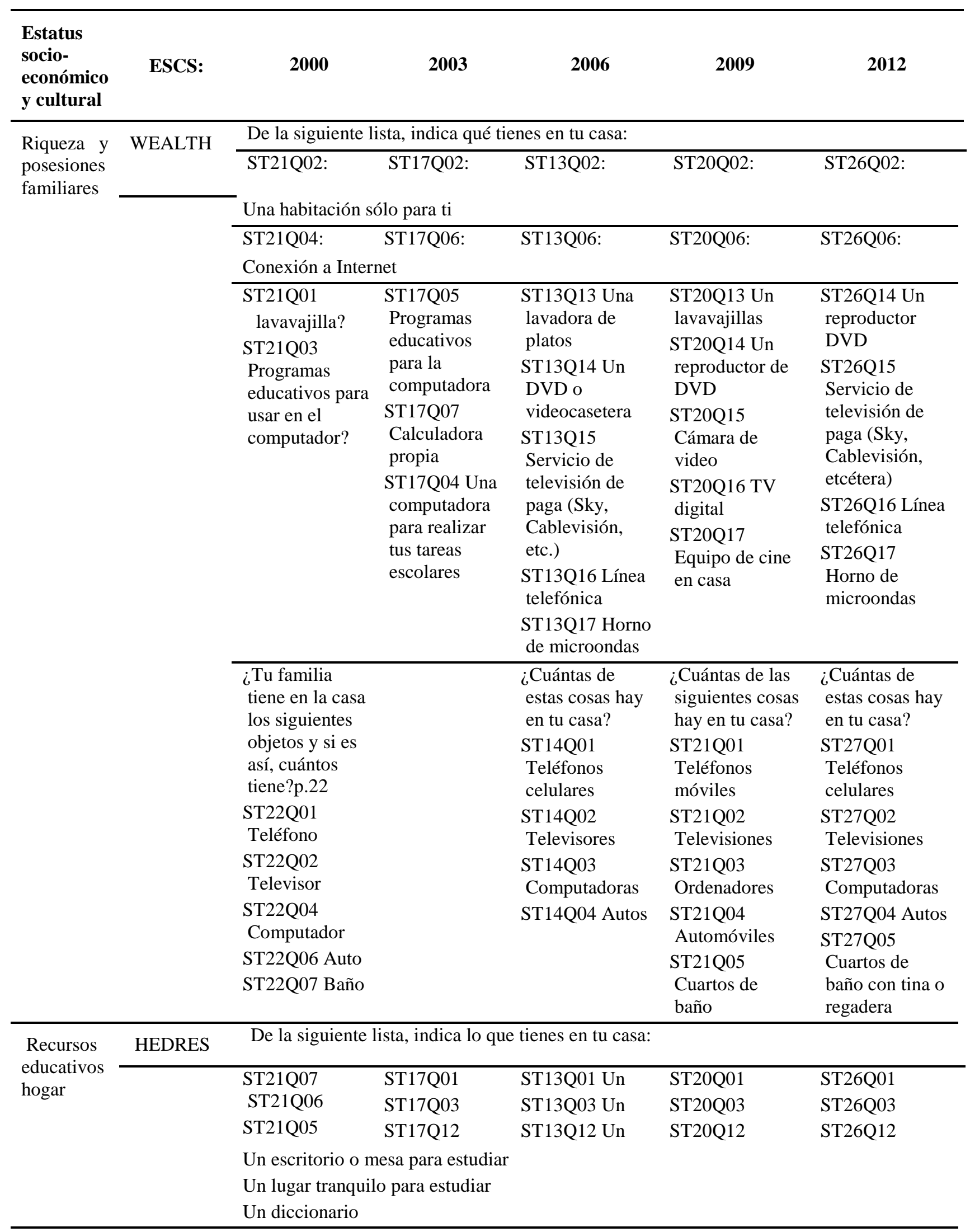


González-Such, José; Sancho-Álvarez, Carlos \& Sánchez-Delgado, Purificación (2016). Cuestionarios de contexto pisa: Un estudio sobre los indicadores de evaluación. RELIEVE, 22(1), M7. DOI: http://dx.doi.org/10.7203/relieve.22.1.8429

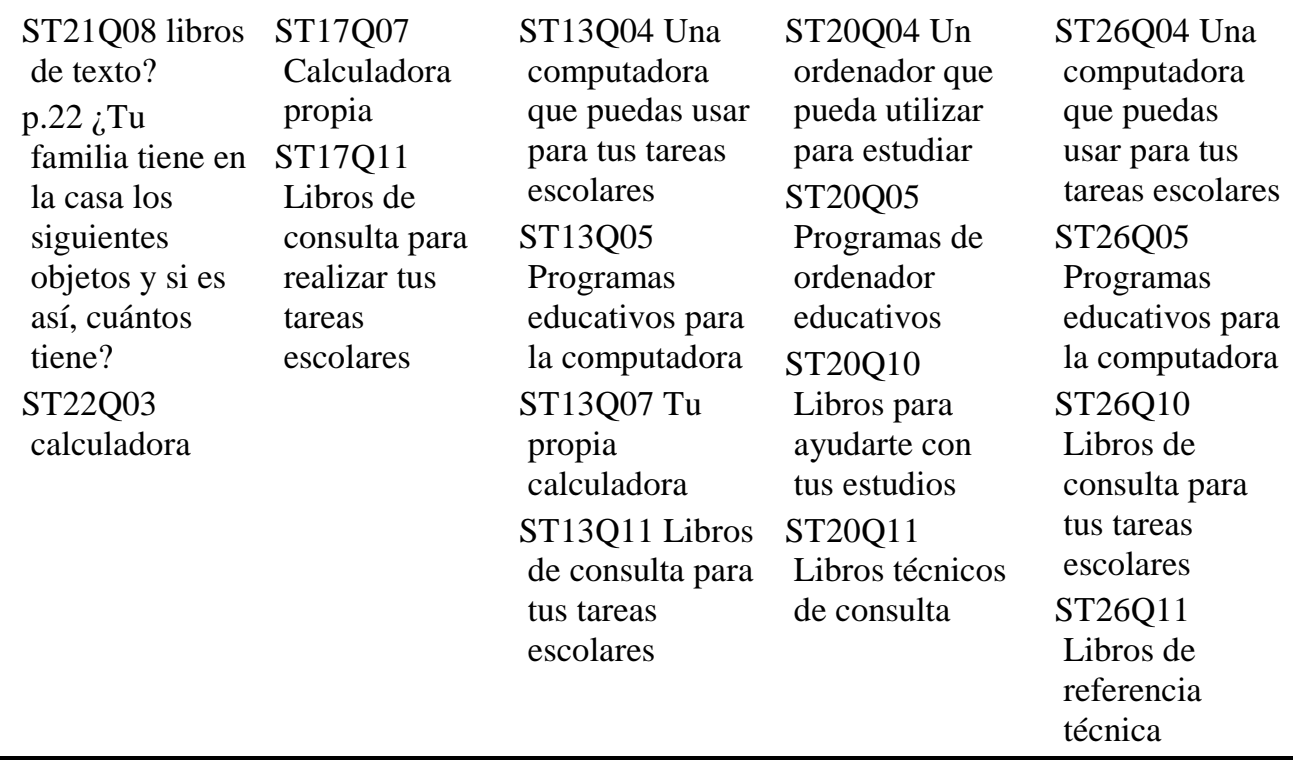

\begin{tabular}{|c|c|c|c|c|c|c|}
\hline \multirow{5}{*}{$\begin{array}{l}\text { Posesiones } \\
\text { culturales }\end{array}$} & \multirow[t]{5}{*}{$\underline{\text { CULTPOSS }}$} & \multicolumn{5}{|c|}{ De la siguiente lista, indica lo que tienes en tu casa: } \\
\hline & & ST21Q09 & ST17Q08 & ST13Q08 & ST20Q07 & ST26Q07 \\
\hline & & ST21Q10 & ST17Q09 & ST13Q09 & ST20Q08 & ST26Q08 \\
\hline & & ST21Q11 & ST17Q10 & ST13Q10 & ST20Q09 & ST26Q09 \\
\hline & & \multicolumn{5}{|c|}{$\begin{array}{l}\text { Libros de literatura clásica (p. ej., El Quijote de Cervantes) } \\
\text { Libros de poesía } \\
\text { Obras de arte (p .ej., pinturas) }\end{array}$} \\
\hline $\begin{array}{l}\text { Posesiones } \\
\text { del hogar }\end{array}$ & HOMEPOS & $\begin{array}{l}\text { WEALTH+ } \\
\text { HEDRES+ } \\
\text { CULTPOSS }\end{array}$ & \multicolumn{4}{|c|}{$\begin{array}{l}\text { WEALTH+ } \\
\text { HEDRES+ } \\
\text { CULTPOSS+ } \\
\text { ¿Cuántos libros hay en tu casa? (ST19Q; ST15; ST22; ST28) }\end{array}$} \\
\hline $\begin{array}{l}\text { Nivel } \\
\text { ocupación } \\
\text { familiar } \\
\text { más alto }\end{array}$ & HISEI & ISEI & \multicolumn{4}{|c|}{ El nivel de ocupación familias más alto de acuerdo a la clasificación ISEI } \\
\hline $\begin{array}{l}\text { Nivel } \\
\text { estudios } \\
\text { familiar } \\
\text { más alto }\end{array}$ & $\begin{array}{l}\text { PARED } \\
\text { (expresado } \\
\text { en años de } \\
\text { escolaridad) }\end{array}$ & ISCED & \multicolumn{4}{|c|}{$\begin{array}{l}\text { El nivel de educación familiar más alto de acuerdo a la clasificación } \\
\text { ISCED }\end{array}$} \\
\hline
\end{tabular}

El caso del ESCS es curioso porque aunque ha ido variando notablemente su cálculo en cuanto a la inclusión de diferencias entre variables para sus indicadores e índice general, a través de los años se ha ido planteando como un único estatus a tener en cuenta para sus análisis. Es decir, como se puede observar entre años, la Riqueza y posesiones familiares no son iguales en ninguna de las oleadas. Así como también podemos observar la misma situación con respecto a los Recursos educativos hogar. Por el contrario, sí existe cierta unificación en relación al indicador Posesiones culturales, Posesiones del hogar, Nivel ocupación familiar más alto y Nivel estudios familiar más alto; destacando que a partir de 2003 se incluye la variable Número de libros hogar.

Por ello, la OCDE ha tenido que ir realizando grandes esfuerzos de compensación entre los cálculos de este polémico índice formado por varios indicadores simples $\mathrm{y}$ 
González-Such, José; Sancho-Álvarez, Carlos \& Sánchez-Delgado, Purificación (2016). Cuestionarios de contexto pisa: Un estudio sobre los indicadores de evaluación. RELIEVE, 22(1), M7. DOI: http://dx.doi.org/10.7203/relieve.22.1.8429

complejos, que sin duda, merece un análisis más pormenorizado y en profundidad; foco de atención que se irá tratando en futuras investigaciones.

En este orden de cosas, es conveniente observar lo que pretende analizar PISA sobre los cuestionarios de contexto 2015. Para ello, se muestra a continuación las medidas con respecto al núcleo de evaluación de contexto y su estructura modular prevista.

Tabla 5. Medidas que deberán incluirse en el núcleo de evaluación de contexto

\begin{tabular}{|c|c|c|c|}
\hline & $\begin{array}{c}\text { CONTEXTO DEL } \\
\text { ESTUDIANTE Y DE LA } \\
\text { ESCUELA }\end{array}$ & PROCESOS & $\begin{array}{l}\text { RESULTADOS NO } \\
\text { COGNITIVOS }\end{array}$ \\
\hline $\begin{array}{c}\text { NIVEL } \\
\text { SISTEMA }\end{array}$ & & $\begin{array}{c}\text { Gobierno: } \\
\text { Toma de decisiones, } \\
\text { diferenciación horizontal y } \\
\text { vertical } \\
\end{array}$ & $\begin{array}{c}\text { (Datos conjuntos del } \\
\text { estudiante) }\end{array}$ \\
\hline $\begin{array}{c}\text { NIVEL } \\
\text { ESCUELA }\end{array}$ & $\begin{array}{c}\text { Localización de la escuela, tipo y } \\
\text { tamaño de la escuela, cantidad y } \\
\text { origen de los recursos (Incl. TIC) } \\
\text { Composición } \\
\text { Social/étnica/académica }\end{array}$ & $\begin{array}{c}\text { Políticas escolares: } \\
\text { Programas ofrecidos, políticas de } \\
\text { admisión y agrupamiento, tiempo } \\
\text { de estudio asignado, soporte al } \\
\text { tiempo de estudio y aprendizaje } \\
\text { adicional, actividades } \\
\text { extracurriculares, desarrollo } \\
\text { profesional, políticas de } \\
\text { evaluación, clima escolar } \\
\text { (conductas del profesor y } \\
\text { estudiante) } \\
\text { Docencia y aprendizaje: } \\
\text { Clima de disciplina, soporte del } \\
\text { profesor, retos cognitivos. }\end{array}$ & $\begin{array}{c}\text { (Datos conjuntos del } \\
\text { estudiante) }\end{array}$ \\
\hline $\begin{array}{c}\text { NIVEL } \\
\text { ESTUDIANTE }\end{array}$ & $\begin{array}{l}\text { Género, estatus socio-económico } \\
\text { (nivel de educación y ocupación } \\
\text { de los padres, posesiones de la } \\
\text { casa número de libros en la casa), } \\
\text { historial de lenguaje y migración, } \\
\text { curso, educación pre-primaria, } \\
\text { edad de entrada en la escuela }\end{array}$ & $\begin{array}{l}\text { Repetición de curso, programas } \\
\text { realizados, tiempo de aprendizaje } \\
\text { en el escuela (lecciones } \\
\text { obligatorias e instrucción } \\
\text { adicional), aprendizaje fuera de la } \\
\text { escuela }\end{array}$ & $\begin{array}{l}\text { Resultados del dominio } \\
\text { general no cognitivo (p.ej. } \\
\text { motivación hacia el } \\
\text { aprendizaje, satisfacción } \\
\text { con la escuela) } \\
\text { Resultados del dominio } \\
\text { específico (motivación, } \\
\text { opiniones y estrategias } \\
\text { relacionadas con el } \\
\text { dominio, } \\
\text { opiniones/creencias } \\
\text { relacionadas con sí mismo, } \\
\text { conducta relacionada con } \\
\text { el dominio) }\end{array}$ \\
\hline
\end{tabular}

Nota: las medidas en cursiva están adaptadas al dominio principal respectivo, p.ej. Ciencias en pisa 2015. Fuente: (OCDE, 2016)

La tabla 6 muestra la estructura modular de PISA 2015, situando los módulos en la estructura general de contexto, proceso y resultados, incluyendo las áreas de contenido de resultados no cognitivos, contexto del estudiante, políticas y gobierno de enseñanza y aprendizaje. 
González-Such, José; Sancho-Álvarez, Carlos \& Sánchez-Delgado, Purificación (2016). Cuestionarios de contexto pisa: Un estudio sobre los indicadores de evaluación. RELIEVE, 22(1), M7. DOI: http://dx.doi.org/10.7203/relieve.22.1.8429

Tabla 6. Estructura modular del diseño de evaluación de contexto PISA 2015

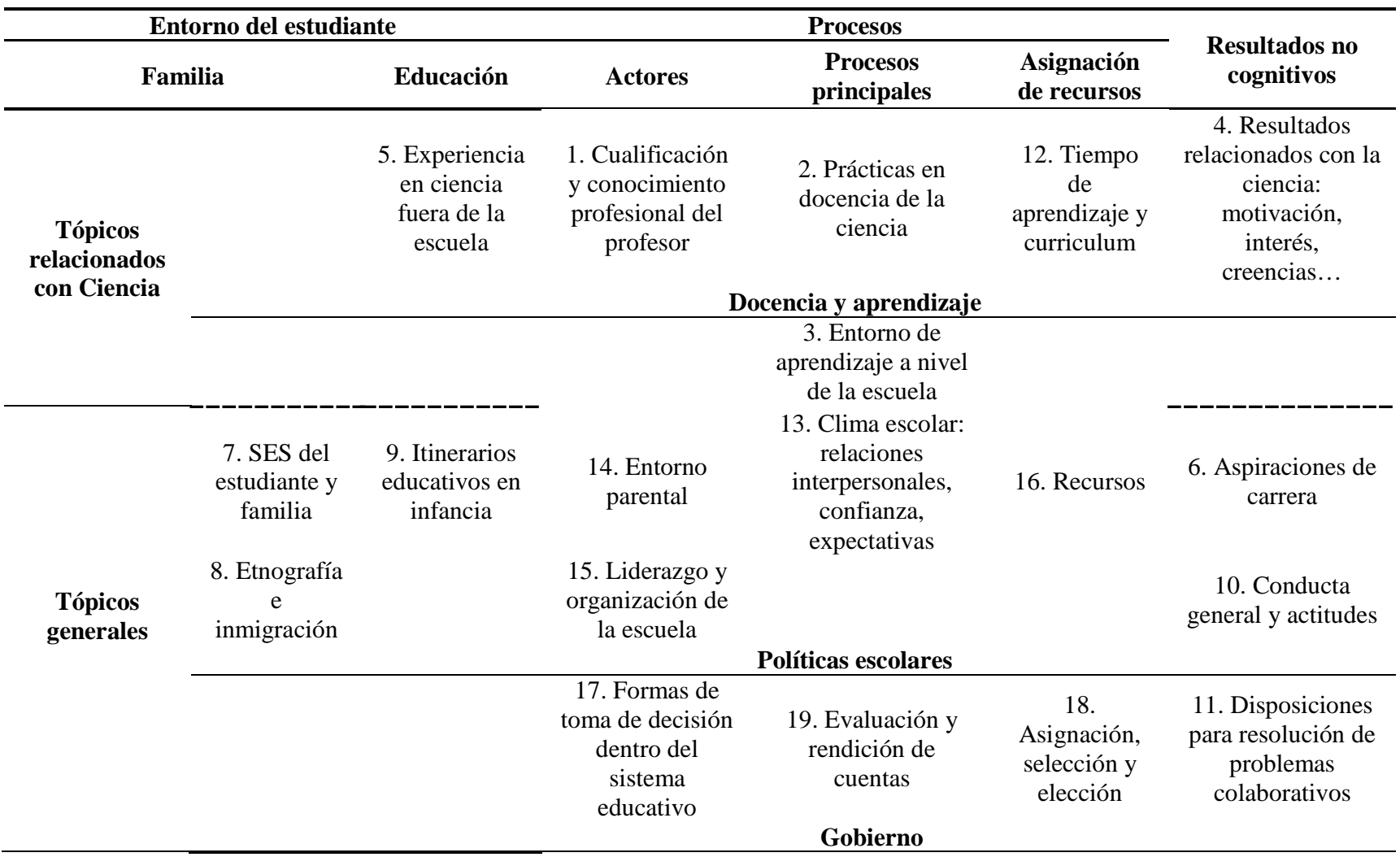

Fuente: (OCDE, 2016, p. 107)

Dentro de los resultados no cognitivos se encuentran los módulos de alta prioridad 10 (Dominio general conducta y actitudes del estudiante) y el 4 (Resultados relacionados con ciencias; motivación, actitudes, creencias) así como los módulos de baja prioridad 6 (carreras de ciencias) y 11 (disposición para la resolución de problemas colaborativa).

Tabla 7. Medidas de resultados no cognitivos incluidas en el cuestionario principal PISA 2015

\begin{tabular}{|c|c|c|}
\hline Area & Relacionado con ciencia (Módulo 4) & Dominio General (Módulos 6, 10, 11) \\
\hline Yo mismo & Auto-eficacia & $\begin{array}{c}\text { Test de ansiedad } \\
\text { Sentirse bien en general (satisfacción vital) } \\
\text { Sentise bien en la escuela (sentido de } \\
\text { pertenencia) }\end{array}$ \\
\hline $\begin{array}{l}\text { Intereses, actitudes y } \\
\text { motivación }\end{array}$ & $\begin{array}{c}\text { Interés en tópicos generales de } \\
\text { ciencias } \\
\text { Disfrute de las ciencias } \\
\text { Motivación instrumental } \\
\end{array}$ & Motivación de rendimiento \\
\hline Creencias y preferencias & $\begin{array}{c}\text { Creencias epistemológicas } \\
\text { Recompensas del entorno } \\
\text { Optimismo del entorno } \\
\end{array}$ & $\begin{array}{l}\text { Colaboración y disposiciones de equipo } \\
\text { Aspiraciones de carrera }\end{array}$ \\
\hline Tecnología-TIC & & $\begin{array}{c}\text { Uso de TIC } \\
\text { Interés en TIC } \\
\text { Competencia percibida en TIC } \\
\text { Autonomía percibida usando TIC } \\
\text { Usos de TIC en interacción social }\end{array}$ \\
\hline Conducta & & $\begin{array}{c}\text { Salud: Actividades físicas } \\
\text { Uso del tiempo: actividades antes/después de la } \\
\text { escuela }\end{array}$ \\
\hline
\end{tabular}

Nota: Negrita: medidas de tendencia. Fuente: OCDE (2016, p. 109) 
Otro apartado considerado es el de la evaluación de los procesos de enseñanza aprendizaje, con los módulos de alta prioridad 2 (prácticas docentes de ciencias), 12 (aprendizaje y curriculum) y 1 (cualificaciones de profesor y conocimiento profesional), junto con los del módulo de baja prioridad 5 (experiencia fuera de la escuela en ciencias).

Tabla 8. Evaluación del tiempo de aprendizaje y pérdida del tiempo de aprendizaje en PISA 2012

\begin{tabular}{|c|c|c|c|c|}
\hline & & $\begin{array}{l}\text { Cuestionario de } \\
\text { estudiante }\end{array}$ & $\begin{array}{c}\text { Cuestionario de } \\
\text { escuela }\end{array}$ & \\
\hline Uso & Estudiante & $\begin{array}{c}\text { + Instrucción adicional } \\
\text { y tiempo de estudio (uso } \\
\text { del tiempo) } \\
\text { - Absentismo }\end{array}$ & & $\begin{array}{l}\text { Tiempo de vinculación }(\mathrm{ET})=\mathrm{RT}- \\
\text { Absentismo del estudiante, absentismo } \\
\text { escolar, tiempo no vinculado }\end{array}$ \\
\hline \multirow[b]{2}{*}{ Provisión } & Aula & $\begin{array}{l}\text { - Clima de disciplina y } \\
\text { pérdidas en las clases de } \\
\text { ciencias }\end{array}$ & & $\begin{array}{c}\text { Tiempo de aprendizaje realizado }(\mathrm{RT})= \\
\text { PT - pérdidas debidas a organización de la } \\
\text { clase, tiempo de evaluación, tiempos de } \\
\text { espera, etc. }\end{array}$ \\
\hline & Escuela & $\begin{array}{l}\text { + Cantidad de tiempo de } \\
\text { aprendizaje de la } \\
\text { escuela } \\
\text { +Número y tipo de } \\
\text { clases de ciencias }\end{array}$ & $\begin{array}{l}\text { Pérdidas en el } \\
\text { nivel Escuela }\end{array}$ & $\begin{array}{c}\text { Tiempo de aprendizaje proporcionado (PT) } \\
\text { = AT - pérdidas debidas al tiempo, } \\
\text { vacaciones, absentismo del profesorado, } \\
\text { etc. }\end{array}$ \\
\hline
\end{tabular}

Fuente: OCDE (2016, p. 113)

Finalmente, las medidas relacionadas con el profesor se muestran en la Tabla 9.

Tabla 9. Medidas relacionadas con el profesor en la prueba de campo PISA 2015

\begin{tabular}{|c|c|c|}
\hline & Relacionados con las ciencias & General \\
\hline Contexto & \multicolumn{2}{|c|}{ Género, edad, estatus de empleo, experiencia laboral, materias estudiadas } \\
\hline \multirow[t]{2}{*}{$\begin{array}{c}\text { Educación } \\
\text { inicial }\end{array}$} & \multicolumn{2}{|c|}{$\begin{array}{c}\text { Objetivo de la primera titulación, tipo de educación docente y programa de } \\
\text { aprendizaje (si lo hay), modo de titulación } \\
\text { Número de profesores por nivel educativo (ScQ) }\end{array}$} \\
\hline & \multicolumn{2}{|c|}{$\begin{array}{c}\text { Contenido relacionado con las ciencias } \\
\text { Número de profesores de ciencias por nivel de titulación (ScQ) }\end{array}$} \\
\hline \multirow[t]{2}{*}{$\begin{array}{l}\text { Desarrollo } \\
\text { profesional }\end{array}$} & \multicolumn{2}{|c|}{$\begin{array}{l}\text { Participación en distintos tipos de actividades } \\
\text { Cantidad de participación obligada, políticas escolares (ScQ) }\end{array}$} \\
\hline & $\begin{array}{c}\text { Colaboración } \\
\text { Contenido relacionado con las ciencias } \\
\end{array}$ & $\begin{array}{c}\text { Cooperación } \\
\text { Contenido general } \\
\end{array}$ \\
\hline Creencias & $\begin{array}{l}\text { Autoeficacia ( relacionado con el contenido en ciencias y la } \\
\text { docencia en ciencias) }\end{array}$ & $\begin{array}{c}\text { Satisfacción } \\
\text { laboral }\end{array}$ \\
\hline
\end{tabular}

Nota: si no se indica lo contrario, los constructos están incluidos en el Cuestionario del Profesor opcional PISA 2015. Fuente: (OCDE, 2016, p. 114)

Finalmente, en el apartado de Políticas de evaluación, asesoramiento y gobierno, se incluyen el módulo 19 (asesoramiento, evaluación y rendición de cuentas) de alta prioridad y los módulos de baja prioridad 3 (entorno de aprendizaje a nivel de escuela para las ciencias) y 13-18 -ver tabla 10. 
Tabla 10. Medidas en PISA 2015 relacionadas con evaluación y rendición de cuentas

\begin{tabular}{|c|c|c|c|c|}
\hline & $\begin{array}{c}\text { Evaluación } \\
\text { externa }\end{array}$ & $\begin{array}{c}\text { Evaluación del } \\
\text { profesor }\end{array}$ & $\begin{array}{c}\text { Evaluación } \\
\text { interna }\end{array}$ & Evaluación formativa \\
\hline \multirow{2}{*}{$\begin{array}{l}\text { Fines y } \\
\text { criterios }\end{array}$} & \multicolumn{4}{|c|}{$\begin{array}{c}\text { Práctica general de la evaluación (ScQ) } \\
\text { Fin de los resultados de evaluación (ScQ) }\end{array}$} \\
\hline & \multicolumn{2}{|c|}{ Políticas de evaluación (ScQ) } & & $\begin{array}{c}\text { Calificaciones del } \\
\text { Profesor (TQG) }\end{array}$ \\
\hline Prácticas & & $\begin{array}{c}\text { Métodos de } \\
\text { evaluación del } \\
\text { profesor (ScQ) } \\
\end{array}$ & & $\begin{array}{c}\text { Instrumentos de } \\
\text { evaluación en el aula } \\
\text { (TQC/TALIS) }\end{array}$ \\
\hline $\begin{array}{c}\text { Usos y } \\
\text { consecuencias }\end{array}$ & $\begin{array}{c}\text { Procesos de } \\
\text { evaluación } \\
\text { externa (ScQ) } \\
\text { Usos de los datos } \\
\text { de logro para } \\
\text { rendición de } \\
\text { cuentas (ScQ) }\end{array}$ & & $\begin{array}{l}\text { Consecuencias de } \\
\text { la evaluación } \\
\text { interna (ScQ) }\end{array}$ & $\begin{array}{l}\text { Retroalimentación: } \\
\text { percepción del } \\
\text { estudiante (StQ) } \\
\text { Adaptación de la } \\
\text { instrucción (StQ) }\end{array}$ \\
\hline
\end{tabular}

Fuente: OCDE (2016, p. 116)

\section{Estudios sobre indicadores}

Los estudios en los que se utilizan estos indicadores se centran en la relación existente entre una o más variables del tipo Rendimiento, Género, Comunidades Autónomas e indicadores simples o compuestos como los reportados por PISA in Focus (de carácter general http://www.mecd.gob.es/inee/PISA-infocus.html) 0 en algunos de boletines EducaINEE

(http://www.mecd.gob.es/inee/Boletin-deeducacion.html) en los que se extraen resultados relevantes de estudios PISA en general o centrados en determinados países, en nuestro caso en España, en algunos casos analizando las diferencias por Comunidades Autónomas, como en el caso de la serie de CCAA.

Algunos de los resultados han sido: 
Tabla 11. Resumen de resultados de algunas investigaciones con indicadores de contexto

\begin{tabular}{|c|c|c|c|}
\hline FECHA & TÍTULO & RESULTADOS & INDICADORES \\
\hline JUN-15 & $\begin{array}{l}\text { Asistencia a la } \\
\text { Educación } \\
\text { Infantil y } \\
\text { Rendimiento en } \\
\text { Matemáticas. El } \\
\text { caso de las } \\
\text { CC.AA. } \\
\text { españolas } \\
\text { (INEE, 2015a) } \\
\end{array}$ & $\begin{array}{l}\text { Los estudiantes que asistieron a Educación Infantil muestran un rendimiento superior } \\
\text { en matemáticas que aquellos que no lo hicieron. Esta diferencia es significativa en } \\
\text { todas las CC.AA. }\end{array}$ & $\begin{array}{l}\text { A mayor Î́ndice de Estatus Económico, Social y Cultural } \\
\text { de PISA mayor probabilidad de acceder a la Educación } \\
\text { Infantil por CCAA }\end{array}$ \\
\hline DIC-14 & $\begin{array}{l}\text { Motivación para } \\
\text { aprender } \\
\text { matemáticas y } \\
\text { PISA 2012: el } \\
\text { caso de las } \\
\text { CC.AA. } \\
\text { españolas } \\
\text { (INEE, 2014a) } \\
\end{array}$ & $\begin{array}{l}\text { En España, los estudiantes que están más motivados para aprender matemáticas, } \\
\text { porque consideran que será beneficioso para sus futuros estudios y carreras, obtienen } \\
\text { mejor puntuación en matemáticas. }\end{array}$ & $\begin{array}{l}\text { En promedio, los chicos están más motivados para } \\
\text { aprender matemáticas que las chicas. En las CC.AA., esta } \\
\text { brecha de género en la motivación está asociada } \\
\text { positivamente con la diferencia de puntuación en } \\
\text { matemáticas entre chicos y chicas. CCAA }\end{array}$ \\
\hline JUN-14 & $\begin{array}{l}\text { Perseverando } \\
\text { hacia el éxito en } \\
\text { los estudios: } \\
\text { PISA } 2012 \text { y las } \\
\text { CC.AA. (INEE, } \\
\text { 2014b) }\end{array}$ & $\begin{array}{l}\text { Los estudiantes españoles manifiestan unos niveles de perseverancia que se encuentran } \\
\text { entre los más elevados de los países de la OCDE. Todas las comunidades que han } \\
\text { participado en PISA } 2012 \text { superan la media de los países desarrollados, salvo Illes } \\
\text { Balears y Cataluña. Andalucía, Madrid, País Vasco y Extremadura destacan por lo } \\
\text { elevado de sus índices de perseverancia }\end{array}$ & $\begin{array}{l}\text { Los datos indican la existencia de un efecto positivo de la } \\
\text { perseverancia sobre la puntuación. } \\
\text { PISA } 2012 \text { plantea a los estudiantes preguntas específicas } \\
\text { acerca de si ante la aparición de un problema se rinden } \\
\text { enseguida; posponen los problemas difíciles; o por el } \\
\text { contrario permanecen interesados en las tareas que } \\
\text { empiezan y siguen trabajando en una tarea hasta que todo } \\
\text { está perfecto. CCAA }\end{array}$ \\
\hline MAY-14 & $\begin{array}{l}\text { Ocupaciones de } \\
\text { los padres y } \\
\text { PISA } 2012 \\
\text { (INEE, 2014c) }\end{array}$ & $\begin{array}{l}\text { Existen diferencias sustanciales en el rendimiento educativo de los estudiantes en } \\
\text { función del tipo de trabajo de los padres. En el ámbito internacional, los hijos de padres } \\
\text { con ocupaciones más cualificadas tienden a obtener mejores resultados que el resto de } \\
\text { estudiantes. Los sistemas educativos de las comunidades autónomas con una } \\
\text { composición del empleo más orientada a las ocupaciones más cualificadas, obtienen } \\
\text { mejores puntaciones medias. }\end{array}$ & Ocupación padres CCAA \\
\hline MAR-14 & $\begin{array}{l}\text { Hacer novillos y } \\
\text { PISA } 2012 \\
\text { (INEE, 2014d) }\end{array}$ & $\begin{array}{l}\text { En PISA } 2012 \text { los estudiantes fueron preguntados acerca de cuántas veces habían } \\
\text { llegado tarde o faltado a alguna clase o a días enteros de colegio sin autorización } \\
\text { durante las dos semanas previas a la prueba. En el conjunto de la OCDE el 35\% llegó } \\
\text { tarde alguna vez al centro, porcentaje idéntico al de España. Existen diferencias de } \\
\text { hasta } 15 \text { puntos entre las CC. AA. participantes en PISA. } \\
\text { Cuanto más elevado es el porcentaje de alumnos que faltan días enteros de clase, }\end{array}$ & Pregunta llegar tarde o faltar. Rendimiento por CCAA \\
\hline
\end{tabular}


González-Such, José; Sancho-Álvarez, Carlos \& Sánchez-Delgado, Purificación (2016). Cuestionarios de contexto pisa: Un estudio sobre los indicadores de evaluación. RELIEVE, 22(1), M7. DOI: http://dx.doi.org/10.7203/relieve.22.1.8429

\begin{tabular}{|c|c|c|c|}
\hline FEB-16 & $\begin{array}{l}\text { Alumnos de } \\
\text { bajo } \\
\text { rendimiento: por } \\
\text { qué se quedan } \\
\text { atrás y cómo se } \\
\text { les puede ayudar } \\
\text { (INEE, 2016) }\end{array}$ & $\begin{array}{l}\text { El estudio PISA define a los estudiantes con bajo rendimiento como aquéllos cuya } \\
\text { puntuación está debajo del nivel } 2 \text { en la escala de PISA. }\end{array}$ & $\begin{array}{l}\text { Rendimiento / PIB / Repetir curso / deberes / Asignaturas } \\
\text { / Sexo / Faltas de asistencia / Perseverancia / Liderazgo / } \\
\text { Recursos }\end{array}$ \\
\hline JUN-15 & $\begin{array}{l}\text { Efectos de los } \\
\text { compañeros de } \\
\text { clase en el } \\
\text { rendimiento } \\
\text { académico } \\
\text { (INEE, 2015b) }\end{array}$ & $\begin{array}{l}\text { Un aumento de } 10 \text { puntos porcentuales en la proporción de niñas mejora los resultados } \\
\text { académicos generales y en mátemáticas de niños. Sobre resultado de niñas no es } \\
\text { significativo, }\end{array}$ & Composición de género por las clases / Rendimiento \\
\hline NOV-14 & $\begin{array}{l}\text { Los resultados } \\
\text { de aprendizaje } \\
\text { en matemáticas } \\
\text { en PISA } 2012 \\
\text { (INEE, 2014e) }\end{array}$ & $\begin{array}{l}\text { - El factor más importante a la hora de explicar diferencias en los resultados de } \\
\text { matemáticas es el de la repetición. La diferencia de rendimiento entre los estudiantes } \\
\text { que han repetido alguna vez y los no repetidores corresponde a más de dos años de } \\
\text { escolarización. Existe una relación inversa entre el nivel socioeconómico y el } \\
\text { porcentaje de estudiantes repetidores, hecho que cuestiona la equidad del sistema. } \\
\text { - Por otro lado, como se recoge en otros informes de PISA, la educación en un centro } \\
\text { público o privado apenas influye en los resultados de matemáticas. } \\
\text { - Tanto el esfuerzo como la productividad son factores importantes para explicar los } \\
\text { resultados académicos. }\end{array}$ & $\begin{array}{l}\text { Indice de Estatus Sociométrico y Cultural (ESCS) / } \\
\text { Centro (Pub,priv) / repetición curso (Sí/No) } \\
\text { Productividad / Esfuerzo }\end{array}$ \\
\hline JUL-14 & $\begin{array}{l}\text { Los resultados } \\
\text { españoles en la } \\
\text { competencia } \\
\text { financiera en } \\
\text { PISA (I) (INEE, } \\
\text { 2014f) }\end{array}$ & $\begin{array}{l}\text { • El nivel de competencia financiera de los estudiantes españoles se encuentra por } \\
\text { debajo de la media de la OCDE. Ello es debido, principalmente, al menor porcentaje de } \\
\text { alumnos con un nivel alto de competencia financiera. } \\
\text { - Un } 64 \% \text { de la variación de los resultados en competencia financiera en España es } \\
\text { medido por las competencias en matemáticas y lectura. El porcentaje de variación de la } \\
\text { competencia financiera explicado por la competencia en matemáticas es en España el } \\
\text { más elevado de toda la muestra de países evaluados. } \\
\text { • Las chicas españolas obtienen puntuaciones inferiores en competencias financieras } \\
\text { que los chicos, aunque las diferencias no son estadísticamente significativas. } \\
\text { - La condición de inmigrante afecta a las puntuaciones alcanzadas en competencia } \\
\text { financiera en sentido negativo. } \\
\text { • El nivel educativo de los padres influye positivamente en la competencia financiera } \\
\text { de sus hijos. } \\
\text { - Los alumnos que han repetido curso muestran peores resultados que los no } \\
\text { repetidores. La brecha entre estos colectivos es inferior en España que en la OCDE. La } \\
\text { puntuación de los repetidores españoles es superior a la de sus homólogos en la OCDE. } \\
\text { - Municipios más grandes se asocian con mayores puntuaciones en competencia } \\
\text { financiera. }\end{array}$ & $\begin{array}{l}\text { Competencia financiera / Rendimiento / Resolución de } \\
\text { problemas / género / Inmigrante / Repetición / Educación } \\
\text { de los padres / tamaño localidad / competencias en } \\
\text { matemáticas y lectura }\end{array}$ \\
\hline
\end{tabular}

menor tiende a ser la puntuación de los alumnos que no faltan nunca. / Sexo / Faltas de asistencia / Perseverancia / Liderazgo /

rendimiento: por

(INEE, 2016)

académicos generales y en mátemáticas de niños. Sobre resultado de niñas no es

rendimiento

Centro (Pub,priv) / repetición curso (Sí/No)

Productividad / Esfuerzo

porcentaje de estudiantes repetidores, hecho que cuestiona la equidad del sistema.

público o privado apenas influye en los resultados de matemáticas.

- Tanto el esfuerzo como la productividad son factores importantes para explicar los

debajo de la media de la OCDE. Ello es debido, principalmente, al menor porcentaje de alumnos con un nivel alto de competencia financiera.

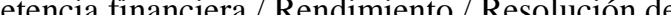

financiera en

medido por las competencias en matemáticas y lectura. El porcentaje de variación de la

financiera en sentido negativo.

de sus hijos.

- Los alumnos que han repetido curso muestran peores resultados que los no

- Municipios más grandes se asocian con mayores puntuaciones en competencia 
González-Such, José; Sancho-Álvarez, Carlos \& Sánchez-Delgado, Purificación (2016). Cuestionarios de contexto pisa: Un estudio sobre los indicadores de evaluación. RELIEVE, 22(1), M7. DOI: http://dx.doi.org/10.7203/relieve.22.1.8429

MAY-14 Ordenadores y Existe una moderada evidencia sobre el efecto positivo del uso de ordenadores en el resultados académicos (INEE, 2014g) conseguir mayor equidad. Sin embargo, los resultados no son del todo significativos, rendimiento escolar en España. En los contextos socioeconómicos más desfavorecidos, el efecto es incluso más significativo, lo que supondría una herramienta potencial para generando dudas acerca del impacto de la utilización de ordenadores sobre el rendimiento académico.

\begin{tabular}{|c|c|}
\hline ABR-14 & $\begin{array}{l}\text { Los resultados } \\
\text { españoles en la } \\
\text { competencia de } \\
\text { resolución de } \\
\text { problemas en } \\
\text { PISA (INEE, } \\
2014 \text { h) }\end{array}$ \\
\hline
\end{tabular}

FEB-14 Análisis desagregado de puntos a favor de los nativos, hasta 57 puntos a favor de quienes no han repetido nunca los resultados de y de hasta 132 puntos a favor de los estudiantes cuando alguno de los padres ha España en completado estudios terciarios. Los resultados apuntan a que si: 1) se estabiliza el PISA (INEE, fenómeno de la inmigración, 2) se consigue una mayor reducción de los problemas de 2014i) repetición de curso, apoyada en una mejora educativa real, y 3) continúa la mejora de los niveles educativos de los padres, cabría esperar en el futuro un progreso adicional de resultados y un mejor posicionamiento de España en el contexto internacional en este ámbito, tan relevante para las posibilidades de desarrollo social y económico.

DIC-13 PISA 2012: resultados por ordenador - Los resultados de España son significativamente más bajos cuando los alumnos realizan la prueba en ordenador en lugar de hacerla en papel.

(INEE, 2013)

- En comparación con lo que sucedía en papel, España presenta una proporción mayo de estudiantes que la OCDE en los niveles inferiores, especialmente en comprensión lectora. En los niveles superiores, la OCDE presenta porcentajes mucho mayores que España, al igual que lo hacía en papel.

- En el conjunto de la OCDE, en matemáticas, ambos sexos obtienen mejores resultados cuando realizan la prueba por ordenador que cuando lo hacen en papel, al contrario que en España. Sin embargo, en comprensión lectora únicamente los chicos obtienen mejores resultados en la prueba por ordenador para el conjunto de la OCDE. Además, las diferencias entre ambos modos de realización de la prueba son mucho mayores en España que en el promedio de la OCDE.

la autonomía en la gestión de los centros, las diferencias entre centros públicos y privados, el uso de los instrumentos de rendición de cuentas o la disciplina en las aulas.

Rendimiento / Uso de ordenadores

Cabras y Tena (2013) en un reciente artículo han estimado el efecto causal del uso de ordenadores en los resultados de los estudiantes españoles en la prueba PISA 2012.

Modelización Bayesiana no Paramétrica (BART) Resolución de problemas / Género / Inmigrantes / Repetición de curso / Estatus sociométrico

El estatus socioeconómico de los alumnos, aproximado en PISA mediante un índice que recoge el nivel educativo de los padres, su ocupación profesional y los recursos tecnológicos y culturales disponibles en el hogar

técnicas shift-share de descomposición de diferencias, el impacto de la condición de inmigrante, de repetidor y del nivel educativo de los padres en la evolución temporal de los resultados obtenidos por España en PISA, así como en la posición relativa respecto al conjunto de países desarrollados

Evaluación de la competencia matemática.

- Evaluación de conocimientos generales y aptitudes relacionadas con las Tecnologías de la Información y la Comunicación (TIC): uso del teclado y el ratón y otras convenciones comunes.

- Evaluación de competencias relacionadas con la interacción entre las matemáticas y las TIC: realización de gráficos a través de un asistente, planificación e implementación de una estrategia de ordenación en una hoja de cálculo para localizar los datos deseados

Centro / Rendimiento / Disciplina 
González-Such, José; Sancho-Álvarez, Carlos \& Sánchez-Delgado, Purificación (2016). Cuestionarios de contexto pisa:

Un estudio sobre los indicadores de evaluación. RELIEVE, 22(1), M7. DOI: $\underline{\text { http://dx.doi.org/10.7203/relieve.22.1.8429 }}$

Por otra parte, en la tabla 12 se muestran algunos estudios sobre PISA 2006 en el que

se utilizan indicadores de contexto.

Tabla 12. Selección de modelos de análisis utilizados en publicaciones sobre PISA 2006 para contextos de logro en ciencias

\begin{tabular}{|c|c|}
\hline Publicación & Pregunta o modelo de investigación \\
\hline Nagengast and Marsh (2014) & Cross-cultural measurement invariance for motivation and engagement in science \\
\hline $\begin{array}{l}\text { Drechsel, Carstensen and Prenzel } \\
\text { (2011) }\end{array}$ & Dimensionality of science interest \\
\hline Olsen and Lie (2011) & Country- and culture specific profiles of interest \\
\hline Ainley and Ainley (2011a) & Students' enjoyment, learning engagement, and achievement \\
\hline Ainley and Ainley (2011b) & Knowledge, affect, value, and students' interest in science \\
\hline Lavonen and Laaksonen (2009) & Learning activities, interest in science, self-efficacy, self-concept, and performance \\
\hline Fensham (2009) & Gender, task context and science performance \\
\hline $\begin{array}{l}\text { Buccheri, Gruber and Bruhwiler } \\
\text { (2011) }\end{array}$ & Gender specificity in interest and vocational choices \\
\hline Mc Conney et al. (2011) & Science interests among minority students \\
\hline Luu and Freeman (2011) & \multirow{2}{*}{ Scientific literacy and ICT-related variables } \\
\hline Kubiatko and Vlckova (2010) & \\
\hline Ho (2010) & Parental involvement and students' science performance \\
\hline Basl (2011) & \multirow{2}{*}{ Explaining interest in future science-related careers } \\
\hline Kjaernsli and Lie (2011) & \\
\hline Willms (2010) & School composition, school and classroom context, and students' literacy skills \\
\hline Dincer and Uysal (2010) & Effects of school programme types \\
\hline Coll et al. (2010) & influence of educational context in a western vs. Asian country \\
\hline
\end{tabular}

Fuente: OCDE (2016, p. 127)

\section{Conclusiones}

Las pruebas PISA se han convertido en un referente mundial para evaluación y mejora de los sistemas educativos de los países que las realizan. A pesar de las opiniones en contra, PISA ha llegado para quedarse. En general se trata de algo abstracto que el público no alcanza a entender pero del que todo el mundo opina.

La necesidad de establecer unos instrumentos de contexto que sitúen a los resultados meramente de rendimiento en su situación real está fuera de toda duda. Resultan especialmente necesarios cuando se trata de evaluar sistemas educativos y de mejorarlos en base a la comparación entre países, como es el fin de PISA.
En este trabajo hemos realizado un repaso sobre los principales indicadores utilizados a través de las distintas ediciones de PISA. A través de este estudio, hemos podido comprobar cómo algunos indicadores han sido utilizados en todas las ediciones, mientras que otros han quedado por el camino, seguramente debido a su poca utilidad.

Se ha visto como algunos indicadores de contexto se han mantenido a lo largo de las distintas ediciones de PISA, mientras que otros han ido variando de simples a complejos y/o desapareciendo. Otros han aparecido en algunas ediciones, desaparecido en las siguientes y vuelto a utilizarse en otras. El establecimiento de un modelo en el que hay unos indicadores que permanecen a lo largo de distintas ediciones permitirá la comparación entre las ediciones y una mejor adecuación de 
los resultados para estudios longitudinales. Este modelo se ha establecido ya en PISA 2015, basándose en la experiencia de PISA 2012.

De la Orden y Jornet (2012) ya señalaron la relevancia de considerar la selección de las variables que medir en cualquier plan de evaluación puesto que ello tiene consecuencias tanto en el enfoque como en los resultados obtenidos. De ahí que destaquen que muchos informes sobre evaluación de sistemas aporten resultados sobre variables de rendimiento de manera aislada respecto a las variables de contexto aludiendo a estos aspectos de manera muy superficial. Por ello, se apuesta por un modelo de evaluación de carácter innovador incorporando el conocimiento adquirido en la investigación educativa, los modelos explicativos del desempeño y una forma de trabajo optimizante para el desarrollo de los sistemas de cuestionarios de contexto.

No cabe duda que el enfoque de indicadores trata de orientar un análisis a nivel macro sobre la educación, por tanto, la información que debemos pedir a este tipo de evaluaciones debe ser a este nivel y no a otros, es decir, ni meso ni micro analítico.

En este sentido, si bien la mayoría de enfoques evaluativos macro sobre indicadores tienen como eje sustantivo los relativos al desempeño del alumnado, tal como señalan Jornet, López-González, \& Tourón (2012) también existe una opción que permite explicar el rendimiento a partir de indicadores denominados contextuales -realmente de entrada, proceso y contexto- (Jornet, 2012). De esta manera, se tendría la posibilidad de dar una información más holista con el fin de identificar las claves para mejorar la educación, o bien dar razón del por qué de determinados resultados, a partir de la relación de las variables de rendimiento con dichas variables de contexto.

La posibilidad de acceder a los datos PISA a los investigadores posibilita la realización de estudios que no se queden en la mera descripción basada en medias o porcentajes, sino que integren en estudios más complejos distintas variables anidadas que sin duda potenciarán el efecto perseguido por PISA.

\section{Referencias}

Adams, R., \& Wu, M. (2002). PISA 2000. Technical Report. París: OECD Publishing.

Bisquerra, R. (2012). Metodología de la investigación educativa. Madrid: La Muralla.

Coleman, J. S., Campbell, E. Q., Hobson, C. J., McPartland, J., Mood, A. M., Weinfeld, F. D., \& York, R. L. (1966). Equality of Educational Opportunity. Washington, DC: US Government Printing Office.

De la Orden, A., \& Jornet, J. M. (2012). La utilidad de las evaluaciones de sistemas educativos: el valor de la consideración del contexto. Bordón, 64(2), 69-88.

Duru-Bellat, M. (2013). Desde el atractivo poder de los datos de PISA a las desilusiones del Benchmarking. ¿Desafío a la evaluación de los sistemas educativos? Profesorado. Revista de currículum y formación del profesorado, 17(2), 94-104. Obtenido de http://recyt.fecyt.es/index.php/profesorado/ar ticle/view/42409/24335

Gaviria, J. L., \& Castro, M. (2005). Modelos jerárquicos lineales. Madrid: La Muralla.

González-Montesinos, Manuel-Jorge \& Backhoff, Eduardo (2010). Validación de un cuestionario de contexto para evaluar sistemas educativos con Modelos de Ecuaciones Estructurales. RELIEVE,16(2), art. 4. doi: http://dx.doi.org/10.7203/relieve.16.2.4133

Instituto Nacional de Evaluación Educativa (INEE). (2013). PISA 2012: resultados por ordenador. EducaINEE (Boletín de educación). Ministerio de Educación, Cultura y Deporte. Obtenido dehttp://www.mecd.gob.es/dctm/inee/boletin es/actualizacion.boletin25/educaineeboletin2 5.pdf?documentId=0901e72b818cf242

Instituto Nacional de Evaluación Educativa (INEE). (2014a). Motivación para aprender matemáticas y PISA 2012: el caso de las CC.AA. españolas. EducaINEE (Boletín de Educación). Ministerio de Educación, 
Cultura y Deporte. Obtenido de http://www.mecd.gob.es/dctm/inee/boletines/ especialccaa/educaineeautonomias4.pdf?doc umentId=0901e72b81b583ae

Instituto Nacional de Evaluación Educativa (INEE). (2014b). Perseverando hacia el éxito en los estudios: PISA 2012 y las CC.AA. EducaINEE (Boletín de educación). Ministerio de Educación, Cultura y Deporte. Obtenido de http://www.mecd.gob.es/dctm/inee/boletines/ especialccaa/educaineeautonomias3.pdf?doc umentId=0901e72b819c7cac

Instituto Nacional de Evaluación Educativa (INEE). (2014c). Ocupaciones de los padres y PISA 2012. EducaINEE (Boletín de educación). Ministerio de Educación, Cultura y Deporte. Obtenido de http://www.mecd.gob.es/dctm/inee/boletines/ especialccaa/educaineeautonomias2.pdf?doc umentId=0901e72b81952125

Instituto Nacional de Evaluación Educativa (INEE). (2014d). Hacer novillos y PISA 2012. EducaINEE (Boletín de educación). Ministerio de Educación, Cultura y Deporte. Obtenido

dehttp://www.mecd.gob.es/dctm/inee/boletin es/especialccaa/educaineeautonomias1.pdf?d ocumentId=0901e72b8190a44e

Instituto Nacional de Evaluación Educativa (INEE). (2014e). Los resultados de aprendizaje en matemáticas en PISA 2012. EducaINEE (Boletín de educación). Ministerio de Educación, Cultura y Deporte. Obtenido de http://www.mecd.gob.es/dctm/inee/boletines/ educainee3925-112014.pdf?documentId=0901e72b81b46542

Instituto Nacional de Evaluación Educativa (INEE). (2014f). Los resultados españoles en la competencia financiera en PISA (I). EducaINEE (Boletín de educación). Ministerio de Educación, Cultura y Deporte. Obtenido de http://www.mecd.gob.es/dctm/inee/boletines/ educainee-35.140709.pdf?documentId=0901e72b81a2be86
Instituto Nacional de Evaluación Educativa (INEE). (2014g). Ordenadores y resultados académicos. EducaINEE (Boletín de educación). Ministerio de Educación, Cultura y Deporte. Obtenido dehttp://www.mecd.gob.es/dctm/inee/boletin es/boletindeeducacion32v3.pdf?documentId= 0901e72b81977c07

Instituto Nacional de Evaluación Educativa (INEE). (2014h). Los resultados españoles en la competencia de resolución de problemas en PISA. EducaINEE (Boletín de educación). Ministerio de Educación, Cultura y Deporte. Obtenido de http://www.mecd.gob.es/dctm/inee/boletines/ educainee-31cba-3-

3.pdf?documentId=0901e72b8190c336

Instituto Nacional de Evaluación Educativa (INEE). (2014i). Análisis desagregado de los resultados de España en PISA. EducaINEE (Boletín de educación). Ministerio de Educación, Cultura y Deporte. Obtenido de http://www.mecd.gob.es/dctm/inee/boletines/ educainee27.pdf?documentId=0901e72b8186 $\underline{8 \mathrm{~b} 0 \mathrm{a}}$

Instituto Nacional de Evaluación Educativa (INEE). (2015a). Asistencia a la Educación Infantil y Rendimiento en Matemáticas. El caso de las CC.AA. españolas. EducaINEE (Boletín de educación), Ministerio de Educación, Cultura y Deporte. Obtenido de http://www.mecd.gob.es/dctm/inee/boletines/ especialccaa/educaineeautonomias5.pdf?doc $\underline{\text { umentId=0901e72b8201fe12 }}$

Instituto Nacional de Evaluación Educativa (INEE). (2015b). Efectos de los compañeros de clase en el rendimiento académico. EducaINEE (Boletín de educación). Ministerio de Educación, Cultura y Deporte. Obtenido de http://www.mecd.gob.es/dctm/inee/boletines/ educainee44.pdf?documentId=0901e72b8201 $\underline{3480}$

Instituto Nacional de Evaluación Educativa (INEE). (2016). Alumnos de bajo rendimiento: por qué se quedan atrás y cómo se les puede ayudar. EducaINEE (Boletín de educación). Ministerio de Educación, Cultura 
y Deporte. Obtenido de http://www.mecd.gob.es/dctm/inee/boletines/ educainee47.pdf?documentId=0901e72b8202 aa4e

Jornet, J. M. (2012). Dimensiones Docentes y Cohesión Social: Reflexiones desde la Evaluación. Revista Iberoamericana de Evaluación Educativa, 5 (1e), pp.349-362. Obtenido

dehttp://www.rinace.net/riee/numeros/vol5num1_e/art27.pdf

Jornet, J. M., López-González, E., \& Tourón, J. (2012). Evaluación de sistemas educativos: teoría y experiencia. Bordón, 64(2), 9-11.

López-González, E., González-Such, J., \& Lizasoain, L. (2012). Explicación del rendimiento a partir del contexto. Algunas propuestas de análisis gráfico y estadístico. Bordón, 64(2), 127-149.

Marchesi, Á. (2006). El informe PISA y la política educativa en España. Revista de Educación, extraordinario, 337-355.

Martínez Arias, R. (2006). La metodología de los estudios PISA. Revista de Educación, extraordinario, 111-129.

Masters, G., \& Wright, B. (1997). The Partial Credit Model. En W. Van del Linden, \& R. Hambleton, Handbook of Modern Item Response Theory. New York/Berlin/Heidelberg: Springer.

OCDE (2002). PISA 2000 Technical Report. Paris: OECD Publishing. Obtenido de https://www.oecd.org/pisa/pisaproducts/3368 8233.pdf

OCDE (2005). PISA 2003. Technical Report. Paris: OECD Publishing. Obtenido de https://www.oecd.org/edu/school/programme forinternationalstudentassessmentpisa/35188 570.pdf

OCDE. (2009). PISA 2006. Technical Report. Paris: OECD Publishing. Obtenido de https://www.oecd.org/pisa/pisaproducts/4202 5182.pdf

OCDE. (2012). PISA 2009. Technical Report. París: OECD Publishing. Obtenido de https://www.oecd.org/pisa/pisaproducts/5003 6771.pdf

OCDE. (2014). PISA 2012. Technical Report. París: OECD Publishing. Obtenido de https://www.oecd.org/pisa/pisaproducts/PIS A-2012-technical-report-final.pdf

OCDE. (2016). PISA 2015 Context Questionnaires Framework. En OECD, PISA 2015 Assessment and Analytical Framework: Science, Reading, Mathematic and Financial Literacy. París: OECD Publishing. doi: http://dx.doi.org/10.1787/9789264255425-7en

Popkewitz, T. (2013). PISA: números, estandarización de la conducta y la alquimia de las materias escolares. Profesorado. Revista de curriculum y formación del profesorado, 17(2), 47-64.

Rasch, G. (1960-1980). Probabilistic models for some intelligence and attainment tests. Copenhge: Nielsen and Lydiche, 1960-1980 (Reeditado en 1980 por University of Chicago Press.

Rendon, S., \& Navarro, E. (2007). Estudio sobre el rendimiento en Matemáticas en España a partir de los datos del informe PISA 2003. Un modelo jerárquico de dos niveles. Revista Iberoamericana sobre Calidad, Eficacia y Cambio en Educación, 5(3), 118136.

Rindermann, H. (2007). The g-factor of international cognitive ability comparisons: the homogeneity of results in PISA, TIMSS, PIRLS and IQ-tests across nations. European Journal of Personality, 21(5), 667-706. doi: http://dx.doi.org/10.1002/per.634

Sancho-Álvarez, C., Jornet, J. M., \& González-Such, J. (2016). El constructo Valor Social Subjetivo de la Educación: validación cruzada entre profesorado de escuela y universidad. Revista de Investigación Educativa, 34(2), 329-350. doi: http://dx.doi.org/10.6018/rie.34.2.226131 


\section{Notas}

[1] 2006 focus in science (JOYSCIE)

[2] 2003 focus in mathematics; 2006 focus in science

[3] 2003 focus in mathematics

[4] 2003 focus in mathematics

[5] 2006 focus in science

[6] 2003 focus in mathematics; 2006 focus in science

[7] 2012 focus at school

[8] 2000 one less

${ }^{[9]} 2003$ one more

${ }^{[10]}$ Every years with variation in the HOMEPOS

[111 2006 focus in science (SCIEACT)

[12] 2009 focus in Read (STIMREAD)

[13] 2009 focus in read (STRSTRAT)

\section{Agradecimientos}

Este trabajo se ha realizado en el marco del proyecto $I+D+I$ Sistema educativo y cohesión social: diseño de un modelo de evaluación de necesidades (SECS/EVALNEC). Ref. EDU2012-37437, financiado por el Ministerio de Economía y Competitividad de España. Así como por Ajudes per a la formació de personal investigador de caràcter predoctoral, en el marc del Subprograma "Atracció de Talent 2013"

\footnotetext{
\begin{tabular}{rcr}
\hline Autor & To know more / Saber más
\end{tabular}

González-Such, José (jose.gonzalez@uv.es).

Pedagogo y Profesor Titular del Departamento de Métodos de Investigación y Diagnóstico en Educación (MIDE) de la Universidad de Valencia. Es el autor de contacto para este artículo. Miembro del Grupo de Evaluación y Medición (GEM). Sus principales líneas de investigación son: Medición y Evaluación Educativa, Evaluación del profesorado e Innovación Educativa. Su dirección postal es: Facultad de Filosofía y Ciencias de la Educación. Universidad de Valencia. Avda. Blasco Ibáñez, 30. 46010-Valencia (España).

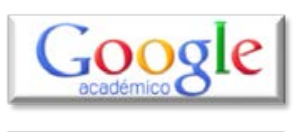

ResearchGate

academia.edu

Autor

To know more / Saber más

Sancho-Álvarez, Carlos (carlos.sancho@uv.es)

Personal investigador en formación del departamento MIDE de la Universitat de València. Máster en Psicopedagogía Social y Comunitaria, y Licenciado en Pedagogía por la Universitat de València, y diplomado en Magisterio por la Universidad de Alcalá. Actualmente realiza el Doctorado en Educación en la Universidad de Valencia. Miembro del Grupo de Evaluación y Medición GEM-Educo. Su dirección postal es: Facultad de Filosofía y Ciencias de la Educación. Universidad de Valencia. Avda. Blasco Ibáñez, 30. 46010-Valencia (España).

\section{Autor}

To know more / Saber más

Sánchez-Delgado, Purificación (purificacion.sanchez@uv.es).

Profesora Doctora del departamento MIDE de la Universitat de València. Doctora en Ciencias de la Educación. Miembro del Grupo de Evaluación y Medición (GEM). En el ámbito de la medición educativa trabaja en el desarrollo y validación de pruebas para medir diferentes tipos de variables educativas vinculadas al área del lenguaje. Su dirección postal es: Facultad de Filosofía y Ciencias de la Educación. Universidad de Valencia. Avda. Blasco Ibáñez, 30. 46010-Valencia (España).
} 
González-Such, José; Sancho-Álvarez, Carlos \& Sánchez-Delgado, Purificación (2016). Cuestionarios de contexto pisa: Un estudio sobre los indicadores de evaluación. RELIEVE, 22(1), M7. DOI:

\section{RELIIEVE}

\section{Revista ELectrónica de Investigación y EValuación Educativa E-Journal of Educational Research, Assessment and Evaluation}

[ISSN: 1134-4032]

(C) Copyright, RELIEVE. Reproduction and distribution of this articles it is authorized if the content is no modified and their origin is indicated (RELIEVE Journal, volume, number and electronic address of the document).

(C) Copyright, RELIEVE. Se autoriza la reproducción y distribución de este artículo siempre que no se modifique el contenido y se indique su origen (RELIEVE, volumen, número y dirección electrónica del documento). 